\title{
Mycobacterial growth inhibition is associated with trained innate immunity
}

\author{
Simone A. Joosten, ${ }^{1}$ Krista E. van Meijgaarden, ${ }^{1}$ Sandra M. Arend, ${ }^{1}$ Corine Prins, ${ }^{1}$ Fredrik Oftung, ${ }^{2}$ Gro Ellen Korsvold, ${ }^{2}$ \\ Sandra V. Kik, ${ }^{3}$ Rob J.W. Arts, ${ }^{4}$ Reinout van Crevel, ${ }^{4}$ Mihai G. Netea, ${ }^{4}$ and Tom H.M. Ottenhoff ${ }^{1}$ \\ 'Department of Infectious Diseases, Leiden University Medical Center, Leiden, Netherlands. 'Division of Infection Control and Environmental Health, Department of Infectious Disease Immunology, \\ Norwegian Institute of Public Health, Oslo, Norway. ${ }^{3}$ KNCV Tuberculosis Foundation, The Hague, Netherlands. ${ }^{4}$ Department of Internal Medicine and Radboud Center for Infectious Diseases, Radboud \\ University Medical Center, Nijmegen, Netherlands.
}

\begin{abstract}
The lack of defined correlates of protection hampers development of vaccines against tuberculosis (TB). In vitro mycobacterial outgrowth assays are thought to better capture the complexity of the human host/Mycobacterium tuberculosis (Mtb) interaction. Here, we used a mycobacterial growth inhibition assay (MCIA) based on peripheral blood mononuclear cells to investigate the capacity to control outgrowth of bacille Calmette-Guérin (BCG). Interestingly, strong control of BCC outgrowth was observed almost exclusively in individuals with recent exposure to Mtb, but not in (long-term) latent TB infection, and only modestly in BCG vaccinees. Mechanistically, control of mycobacterial outgrowth strongly correlated with the presence of a CD14 ${ }^{\mathrm{dim}}$ monocyte population, but also required the presence of T cells. The nonclassical monocytes produced CXCL10, and CXCR3 receptor blockade inhibited the capacity to control BCC outgrowth. Expression of CXCR3 splice variants was altered in recently Mtb-exposed individuals. Cytokines previously associated with trained immunity were detected in MCIA supernatants, and CXCL9, CXCL10, and CXCL11 represent new markers of trained immunity. These data indicate that CXCR3 ligands are associated with trained immunity and are critical factors in controlling mycobacterial outgrowth. In conclusion, control of mycobacterial outgrowth early after exposure to Mtb is the result of trained immunity mediated by a CXCL10producing nonclassical CD14 ${ }^{\text {dim }}$ monocyte subset.
\end{abstract}

\section{Introduction}

Tuberculosis (TB) remains a major threat to human global health, with an estimated one-fourth of the world population latently infected and lifetime reactivation rates between $3 \%$ and $10 \%$ (1). Annually approximately 2 million people die of TB disease, including those suffering from concomitant HIV infection (2). The WHO aims to reduce the burden of TB disease by 2035 (2), and mathematical modeling predicts that successful novel vaccination strategies would be essential to reach that goal $(3,4)$. However, clinical evaluation of new TB vaccines is hampered by the long latency period following infection with Mycobacterium tuberculosis (Mtb) and the low proportion of infected individuals that eventually develop TB disease, thus necessitating large and long-term follow-up studies to determine efficacy against clinical endpoints such as prevention of (or progression to) disease. Production of IFN- $\gamma$ in response to Mtb antigen stimulation has been the gold standard of anti-Mtb immunity. Although IFN- $\gamma$ is required, it is not sufficient for protection against TB disease development $(5,6)$, and preclinical studies even show that high IFN- $\gamma$ can be detrimental such that "more can be worse" (7). Despite intense

Authorship note: SAJ and KEVM contributed equally to this work. Conflict of interest: The authors have declared that no conflict of interest exists. Submitted: September 14, 2017; Accepted: February 13, 2018 Reference information: J Clin Invest. 2018;128(5):1837-1851. https://doi.org/10.1172/JCI97508. research efforts, correlates and mechanisms of (vaccine-induced) protection in TB have remained elusive, hampering rationalized correlate-based down-selection of the expanding list of novel vaccine candidates.

Mycobacterial growth inhibition assays (MGIAs) measure the capacity of whole blood (8) or isolated peripheral blood mononuclear cells (PBMCs) to inhibit the outgrowth of mycobacteria, including bacillus Calmette-Guérin (BCG) (9-13) and Mtb (14). There is a long-standing interest in measuring and understanding the capacity of the immune system to control mycobacterial growth. Several biological assays to quantitate mycobacterial growth have been used over the years, including mycobacteria expressing a reporter (luciferase) $(9,15,16)$. In addition, Hoft and colleagues used primary as well as secondary lymphocyte inhibition assays followed by CFU counting (17-19). Wallis et al. adapted mycobacterial cultures in the BACTEC MGIT system (Becton, Dickinson and Co.) to investigate not only drug sensitivity but also effector immunity $(20,21)$. Over time, several aspects of these different approaches have been integrated into the MGIA assay that is currently used by several groups and includes infection of PBMCs with mycobacteria for several days, followed by incubation in BACTEC MGIT tubes to assess mycobacterial growth capacity $(9,10,12,22)$. Development and optimization of the MGIA assay as it is currently used have been recently reviewed at length (10, 23). MGIA assays have been used already for cells of human ( 9 , $12,24-26)$, nonhuman primate $(26)$, and mouse $(11,13,14)$ origin. 
Although these functional assays may reflect the capacity of individuals to control mycobacterial outgrowth, the underlying mechanism remains unknown. BCG-vaccinated mice have reduced Mtb loads following infection compared with nonvaccinated animals, and this is paralleled by their splenocytes' capacity to reduce mycobacterial outgrowth in MGIA $(11,13)$, supporting the assay's biological validity. BCG vaccination has mostly been used in such studies, because BCG affords at least partial protection against severe manifestations of TB disease, and efficiently induces IFN- $\gamma$-producing T cells $(9,11,13,14,25,26)$. Although many researchers have attempted to identify the cellular subset(s) responsible for mycobacterial growth control, their identity remains inconclusive, and to some extent even elusive. Associations have been found with monocyte/lymphocyte ratios (24), multifunctional $\mathrm{CD}^{+} \mathrm{T}$ cells (25), and iron metabolism (26). Increased iron concentrations, in particular in whole blood-based MGIA assays but also in PBMC assays, significantly promoted the in vitro growth capacity of mycobacteria, such that some caution should be applied to interpreting data from whole blood-based MGIA assays (26).

Mtb infection results in progression to disease in only a small proportion (3\%-10\%) of those infected, while the majority of individuals apparently is able to control mycobacterial outgrowth (2). Recent meta-analyses have shown that latent infection with Mtb (LTBI) results in a striking $79 \%$ protection against reinfection with Mtb, suggesting strong protective host responses induced by Mtb infection (27). Although protection against infection is often thought to be associated with the induction of adaptive immune responses, a recent concept suggests that epigenetic modification of monocytes may also contribute to protection (28). This phenomenon has been termed "trained immunity," and hallmark cytokines produced by these trained monocytes are IL-1 $\beta$, IL-6, and TNF- $\alpha(28,29)$. To explore this biological process and to uncover new biology and corresponding correlates of mycobacterial inhibition, we decided to investigate the capacity of PBMCs from cohorts of BCG vaccinees, as well as individuals with LTBI, patients with active $\mathrm{TB}$, and people from contact investigations following recent TB exposure. PBMC samples with unexpectedly strong control of mycobacterial outgrowth were observed. These were subsequently used to unravel the mechanism of mycobacterial growth control.

\section{Results}

MGIA in BCG cohorts: limited and transient bacterial growth control. Mycobacterial growth inhibition assays (MGIAs) provide an important and hypothesis-free readout of functional (bacterial) growth inhibition by the human immune system. However, in order to apply such functional assays in further research and eventually for rationalized TB vaccine selection, it is essential to optimize the assay toward high reproducibility and robustness. Using a frozen stock of BCG and an extensive thawing procedure, we were able to obtain solidly reproducible standard curves across experiments (Supplemental Figure 1A; supplemental material available online with this article; https://doi.org/10.1172/JCI97508DS1). Serial dilutions were generated as standard curves for each independent experiment, and each standard was plated in a 10-fold dilution series (Supplemental Figure 1D) to obtain CFU counts. Cumula- tive CFU counts over 9 individual experiments were highly reproducible (Supplemental Figure 1B), as were the time-to-positivity (TTP) readouts from the BACTEC over the same dilutions (Supplemental Figure 1C).

BCG vaccination has been widely used for almost a century, but the protection it affords in human populations has been highly variable, likely depending on population geography and previous exposure to environmental factors. We analyzed a cohort of young, mycobacterially naive (purified protein derivative-negative [PPD-negative]) Dutch adults before and at several time points after BCG vaccination (30). We observed considerable variation in mycobacterial growth inhibition between these individuals during follow-up time points, and there appeared no single common time point at which mycobacterial outgrowth across all participants was significantly improved following vaccination (Figure 1A). However, we noticed that several individuals had considerable growth control at 4 weeks after vaccination (left panel), whereas others controlled BCG outgrowth only at 8 (middle panel) or 12 weeks (right panel) after vaccination, suggesting different kinetics in the response (30). Therefore, we compared the prevaccination MGIA result with the results at the peak response for each individual $(4,8$, or 12 weeks after vaccination), and then observed a significant inhibition of mycobacterial outgrowth (Figure 1B). It was remarkable that samples with significant control at 1 time point (e.g., week 4 or 8 ) had lost that capacity at the next sampling time point, which was only 4 weeks later (Figure 1A), suggesting a limited role of imprinted adaptive immunity. Thus, although there was significant BCG growth reduction following BCG vaccination, the magnitude of BCG growth control was rather modest and transient.

LTBI and TB infection: recent exposure associated with strong bacterial growth control. Given the observation that LTBI protects significantly against TB (see above) (27), we decided to assess the capacity of PBMCs from several Mtb-infected cohorts to control BCG outgrowth. We also included TB patients with active disease as well as a control group composed of healthy, mycobacterially naive Dutch donors. Altogether, patients with active TB did not control BCG outgrowth in the MGIA assay (Figure 1C), although we could discriminate 2 subgroups with a different functional response. We have critically analyzed the available clinical parameters of these groups, including disease localization (pulmonary vs. extrapulmonary localization), ethnicity, and suggestion of long-term existing disease, but we were not able to identify any parameter associated with good or poor inhibition of BCG outgrowth.

As only a proportion of latently infected individuals progresses to disease, the capacity to inhibit mycobacterial outgrowth may be expected to characterize the majority of latently infected donors, especially those who have been latently infected for a long period without developing disease. Contrary to our expectation, however, a cohort of latently infected individuals from the Netherlands (31) as well as a group of elderly Norwegian individuals $(32,33)$ known to be latently infected with Mtb for about 3 decades (based on tuberculin skin test conversion and IFN- $\gamma$ release assay positivity) failed to inhibit BCG outgrowth in the MGIA assay (Figure 1, D and E). The LTBI cohort from the Netherlands was composed of individuals with a positive tuberculin skin test (TST), and most individuals also had a positive QuantiFERON-TB Gold (QFN; QIAGEN) response. For most individ- 
A
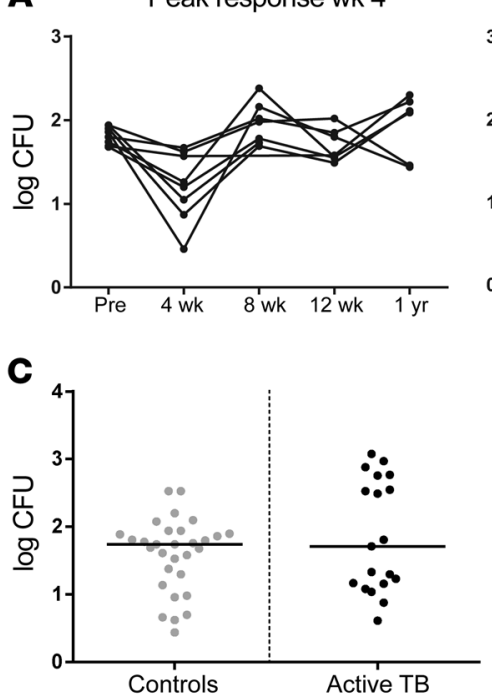

Peak response wk 8

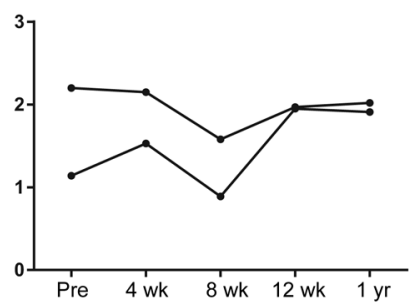

Peak response wk 12

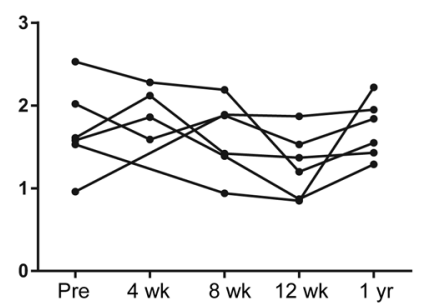

B

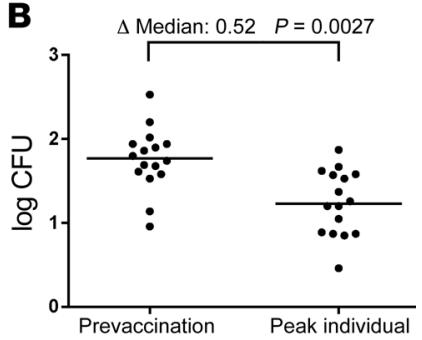

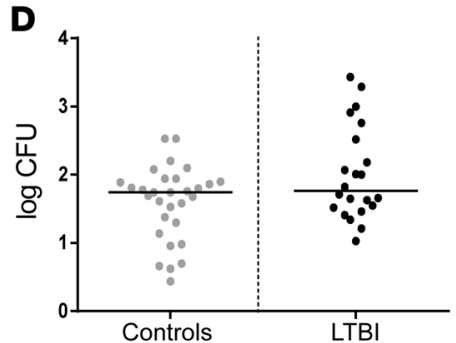
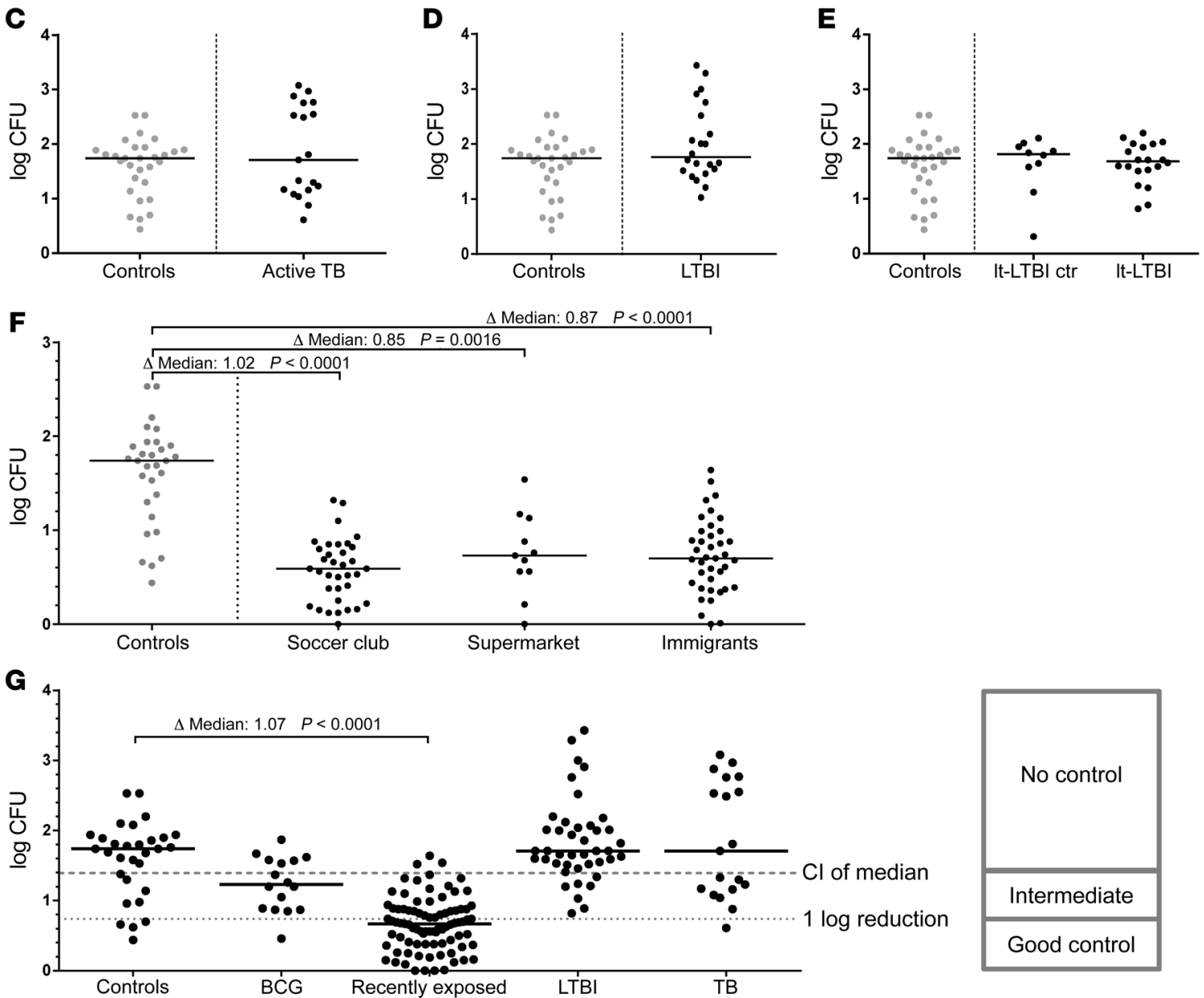

Figure 1. Mycobacterial growth control is associated with recent TB exposure. PBMCs were infected with live BCG, rotated 4 days, and incubated in MGIT tubes in the BACTEC machine. Horizontal lines indicate medians. " $\triangle$ median" indicates the difference in medians between groups. (A) PBMCs collected before BCC vaccination, 4,8 , and 12 weeks after vaccination, and 1 year after vaccination. Left plot indicates donors with the strongest reduction in BCG growth at week $4(n=8)$, middle plot at week $8(n=2)$, and right plot at week $12(n=6)$. (B) For each individual from A, the time point with maximal MGIA control was compared with the prevaccination sample using the Wilcoxon paired-rank test. (C-C) All groups in C-C were compared with a common group of 30 controls - 16 individuals prior to BCC vaccination and 14 blood bank donors - using the Kruskal-Wallis test. (C) Patients with active TB disease ( $n=19)$. (D) Individuals with latent TB infection (LTBI; $n=22$ ). (E) Individuals with long-term LTBI (It-LTBI; $n=20$ ). An age-matched control group (It-LTBI ctr; $n=10)$ was included. (F) Three independent cohorts of recently TB-exposed individuals collected during contact investigations: 35 at a soccer club, 11 in a supermarket, and 39 immigrants with recent exposure to active TB. (C) Combined analysis of all groups tested: BCG ( $n=16$ at peak response); recently exposed (F: $n=85)$; LTBI (from D and E: $n=42$ ); active TB (from C). Data were compared using the Kruskal-Wallis test. Dashed lines indicate the lower limit of the Cl of the median of the controls, and the $1 \mathrm{log}$ reduction compared with this median. Samples above the $\mathrm{Cl}$ of median lacked control of mycobacterial outgrowth and samples with more than 1 log reduction had good control.

uals in our LTBI cohort it is hard to estimate the time since Mtb infection, and since our BCG vaccination studies also revealed a temporal inhibition in BCG growth following vaccination, we hypothesized that mycobacterial growth control might be strongest recently after mycobacterial (TB) exposure.
Next, in order to study this directly, we included samples from 3 fully independent TB contact investigations in the Netherlands: these involved a professional soccer club (34), a supermarket outbreak (35-37), and a group of immigrants with recent (re) exposure to Mtb $(38,39)$. Interestingly, all 3 cohorts considerably 
A

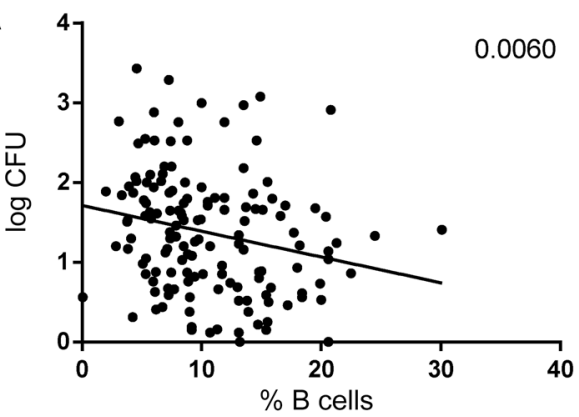

C

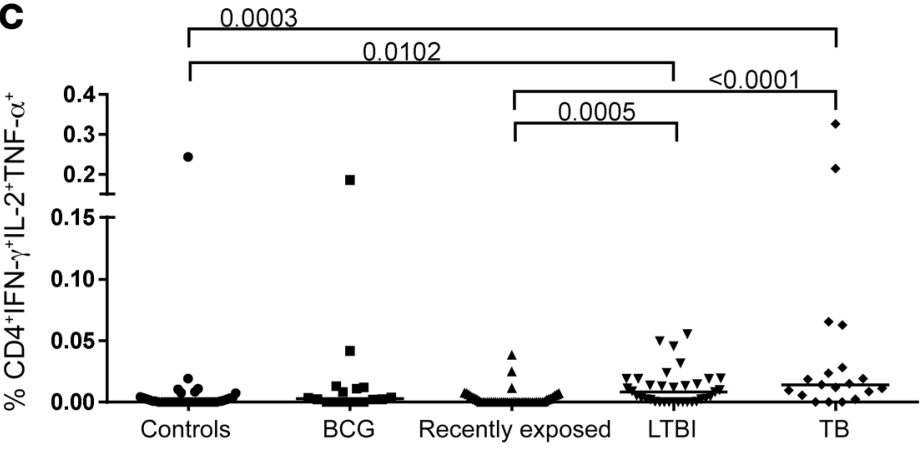

E

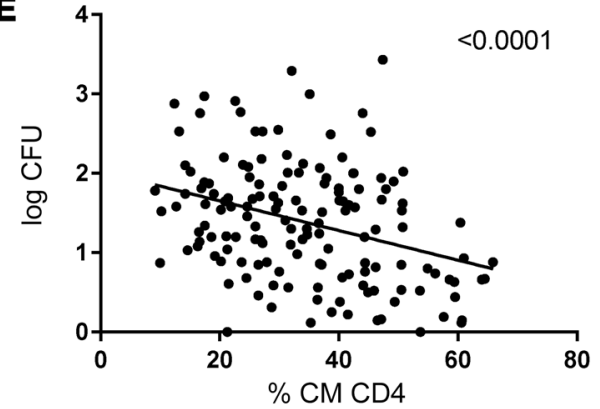

B

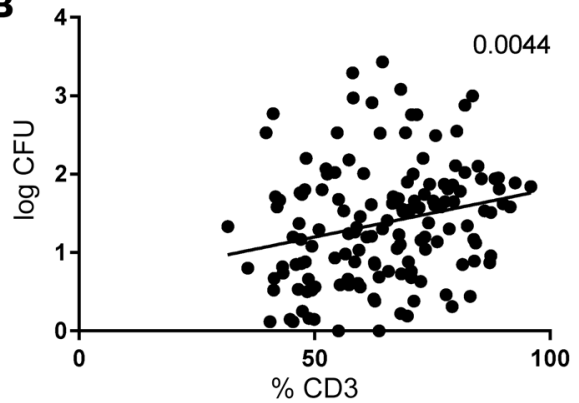

D

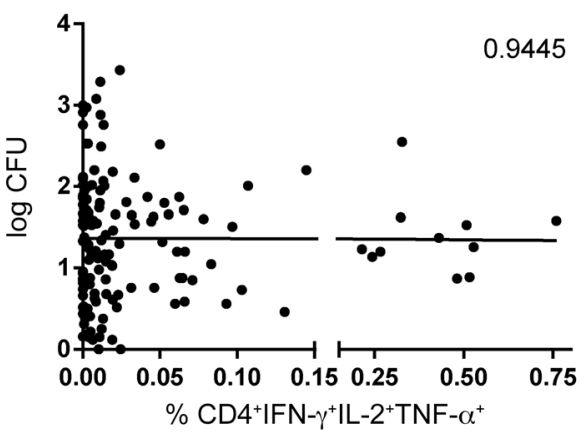

$\mathbf{F}$

\begin{tabular}{|l|c|c|c|}
\cline { 2 - 4 } \multicolumn{1}{c|}{} & $\mathrm{CD}^{+}$ & $\mathrm{CD}^{+}$ & Correlation \\
\hline $\mathrm{N}$ & $\mathrm{NS}$ & $*$ & Positive \\
\hline $\mathrm{CM}$ & $\star \star \star *$ & $\mathrm{NS}$ & Positive \\
\hline $\mathrm{EM}$ & $\mathrm{NS}$ & $\mathrm{NS}$ & \\
\hline $\mathrm{E}$ & $* *$ & $* \star *$ & Inverse \\
\hline
\end{tabular}

Figure 2. Mycobacterial growth control is not associated with multifunctional T cells. PBMCs were infected with live BCC and rotated for 4 days, and samples were incubated in MCIT tubes in the BACTEC machine. Antibody marker staining for FACS analysis was performed after 16 hours of negative control or live BCG stimulation. Data from all independent cohorts were combined. Associations were determined using linear regression modeling $(n=144)$. (A) The percentage of $\mathrm{CD}_{19} \mathrm{~B}^{\mathrm{B}}$ cells was positively correlated with mycobacterial growth control. (B) The percentage of $C D 3^{+} T$ cells was correlated inversely with mycobacterial growth control. (C) The percentage of CD4+ T cells producing IFN- $\gamma$, IL-2, and TNF- $\alpha$ (multifunctional T cells) following 16 hours of BCG stimulation over the different clinical groups. Groups were compared using the Kruskal-Wallis test. Higher numbers were observed in LTBI and TB groups. Controls, $n=38$; BCG, $n=16$; exposed, $n=50$; LTBI, $n=35$; TB, $n=19$. (D) The percentage of CD4+ T cells producing IFN- $\gamma$, IL-2, and TNF- $\alpha$ did not correlate with the capacity to control mycobacterial outgrowth $(n=144)$. (E) The percentage of CD4+ central memory (CM; CD45RA-CCR7 $\left.{ }^{+}\right)$T cells correlated with the capacity to control mycobacterial outgrowth $(n=148)$. (F) Summary of correlation data between $C D 4^{+}$or CD8 ${ }^{+}$T cell subsets based on the expression of CD45RA and CCR7 and the capacity to control mycobacterial outgrowth. Positive correlations indicate that increased expression of the population is associated with better growth control; inverse correlations indicate that increase in the population is associated with decreased growth control. N, naïve; EM, effector memory; $E$, effector.

controlled BCG outgrowth in the MGIA assay (Figure 1F), with a change of about 1 log compared to the median of the control population. To the best of our knowledge, this is the highest level of mycobacterial control reported for human PBMCs so far. Combination of all independent cohorts, previously shown above, according to infectious status, showed that recently exposed individuals inhibited BCG outgrowth best (Figure 1G). Good control, defined as more than $1 \log$ reduction compared with the control population, was almost only observed in donors who were recently exposed. Intermediate control (between the lower confidence interval [CI] value and $1 \log$ reduction of the median of the controls) was observed most frequently in recently exposed individ- uals but also in BCG-vaccinated individuals as well as a subset of TB patients. Taken together, most individuals in the recently exposed group had intermediate or good control, whereas only a small proportion of individuals in the other groups showed such BCG growth inhibition.

The capacity to control BCG outgrowth was compared with clinical measures of Mtb infection status, the TST and QFN, both measures of induced adaptive immunity. Surprisingly, PPD-induced TST induration correlated inversely with the capacity to control BCG outgrowth in the MGIA (Supplemental Figure 2A). However, this was mostly due to the large proportion of individuals that had negative skin test results and relatively good MGIA 
A

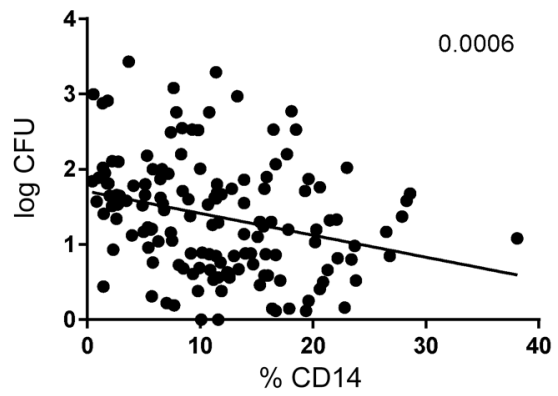

B

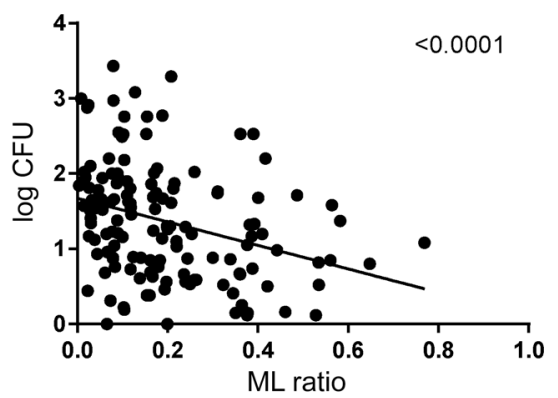

C

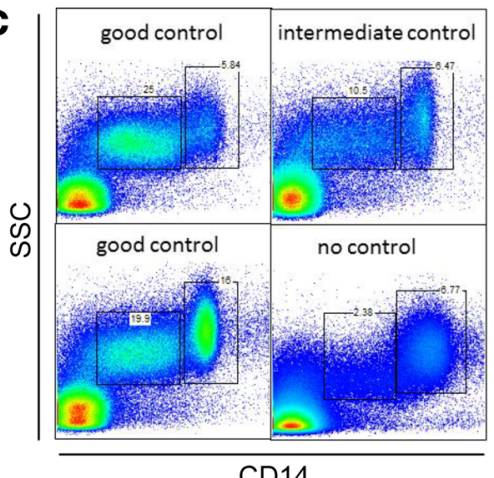

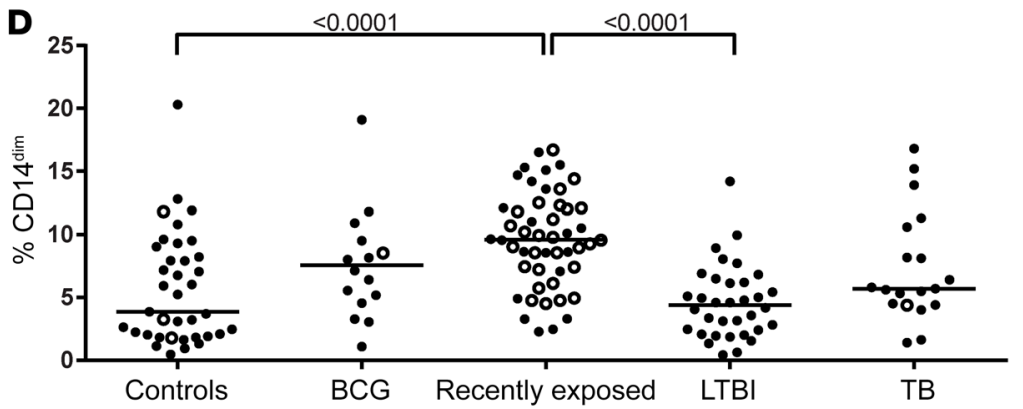
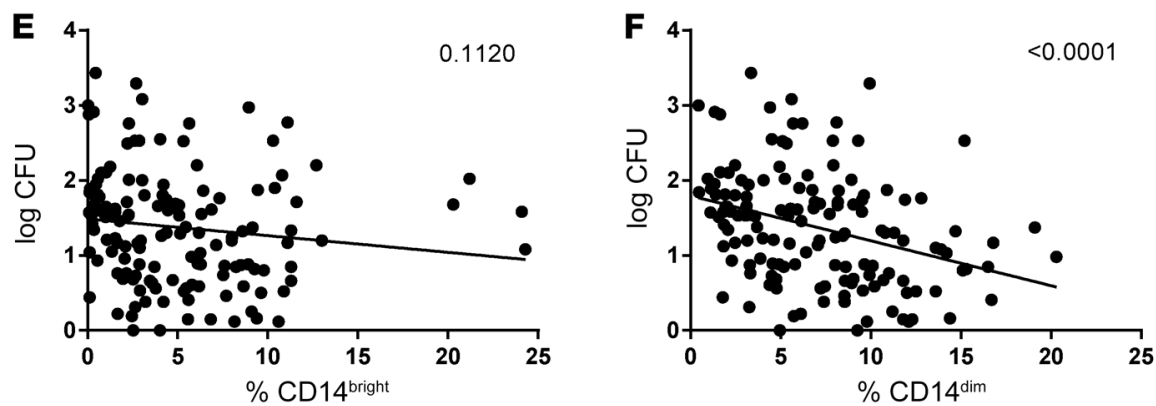

Figure 3. Capacity to control mycobacterial growth is associated with a CD14 ${ }^{\text {dim }}$ myeloid population. PBMCs were infected with live BCC and rotated for 4 days, and samples were incubated in MGIT tubes in the BACTEC machine. Antibody marker staining for FACS analysis was performed after 16 hours of negative control stimulation. Associations were determined using linear regression modeling $(n=144)$. (A) The percentage of CD14 ${ }^{+}$monocytes was positively correlated with mycobacterial growth control. (B) The monocyte/lymphocyte (ML) ratio (\% CD14/CD3) correlated with mycobacterial growth control. (C) Representative dot plots of monocyte profiles in individuals with good mycobacterial growth control (more than 1 log reduction of BCC outgrowth compared with the median of the control population), intermediate control (between 1 log reduction and lower limit of the $\mathrm{Cl}$ of median of control population; see Figure 1G), or no control (above lower limit of the $\mathrm{Cl}$ of median of control population). There was a strong CD14 dim population in the individuals with mycobacterial growth control. (D) Recently exposed individuals had the highest proportion of CD14 ${ }^{\text {dim }}$ cells; open symbols indicate donors with good control in the MGIA. Controls, $n=37$; BCG, $n=16$; exposed, $n=50$; LTBI, $n=34$; TB, $n=19$. (E) The percentage of CD14 bright monocytes was not correlated with mycobacterial growth control $(n=144)$. $(\mathbf{F})$ The percentage of CD14 dim monocytes was positively correlated with mycobacterial growth control $(n=144)$.

control. If these individuals were excluded from the analysis, the negative correlation was lost. There also was no positive correlation between the absolute QFN result and BCG growth control (Supplemental Figure 2B). However, a considerably sized group of individuals lacked cellular immunity toward Mtb antigens while having good control of BCG outgrowth in the MGIA.

Immune correlate analysis: CD14 $4^{\text {dim }}$ monocytes correlate with MGIA control. To decipher the mechanism of mycobacterial growth control mediated mostly by the samples from recently exposed individuals, we analyzed associations with several immune cell subsets and functions. The percentage of $\mathrm{CD} 19^{+}$ B cells correlated with the capacity to control BCG in the MGIA, as higher proportions of $\mathrm{B}$ cells were associated with decreased $\mathrm{CFU}$ (Figure 2A). In contrast, the percentage of $\mathrm{CD}^{+} \mathrm{T}$ cells was inversely related to control in the MGIA (Figure 2B). Since it was previously reported that multifunctional $\mathrm{T}$ cells were associated with MGIA results (25), we also enumerated multifunctional $\mathrm{T}$ cells in our samples (gating strategies are given in Supplemental Figure 7, A and B). However, the frequency of $\mathrm{CD}^{+} \mathrm{T}$ cells producing IFN- $\gamma$, TNF- $\alpha$, and IL-2 was very low in our cohorts, although significant differences were identified (Figure 2C). The same was true for $\mathrm{CD}^{+}$multifunctional $\mathrm{T}$ cells (Supplemental Figure 3A). There was no correlation between the frequency of $\mathrm{CD}^{+}$multifunctional T cells and the MGIA results (Figure 2D), nor was there for $\mathrm{CD}^{+}$multifunctional $\mathrm{T}$ cells (Supplemental Figure 3B). In addition, none of the single-cytokine-producing $\mathrm{CD} 4^{+}$ or $\mathrm{CD}^{+} \mathrm{T}$ cells (Supplemental Figure 3, $\mathrm{C}$ and $\mathrm{D}$ ) was associated with MGIA activity (Supplemental Figure 3, E and F). 
A

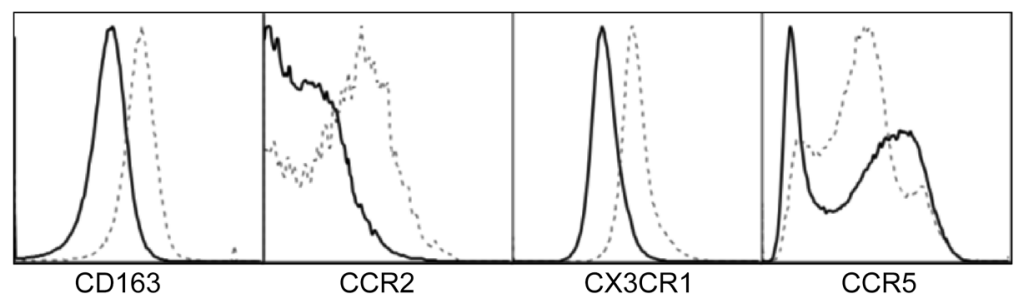

CD14dim
$-\ldots \operatorname{CD} 14^{\text {brigh }}$
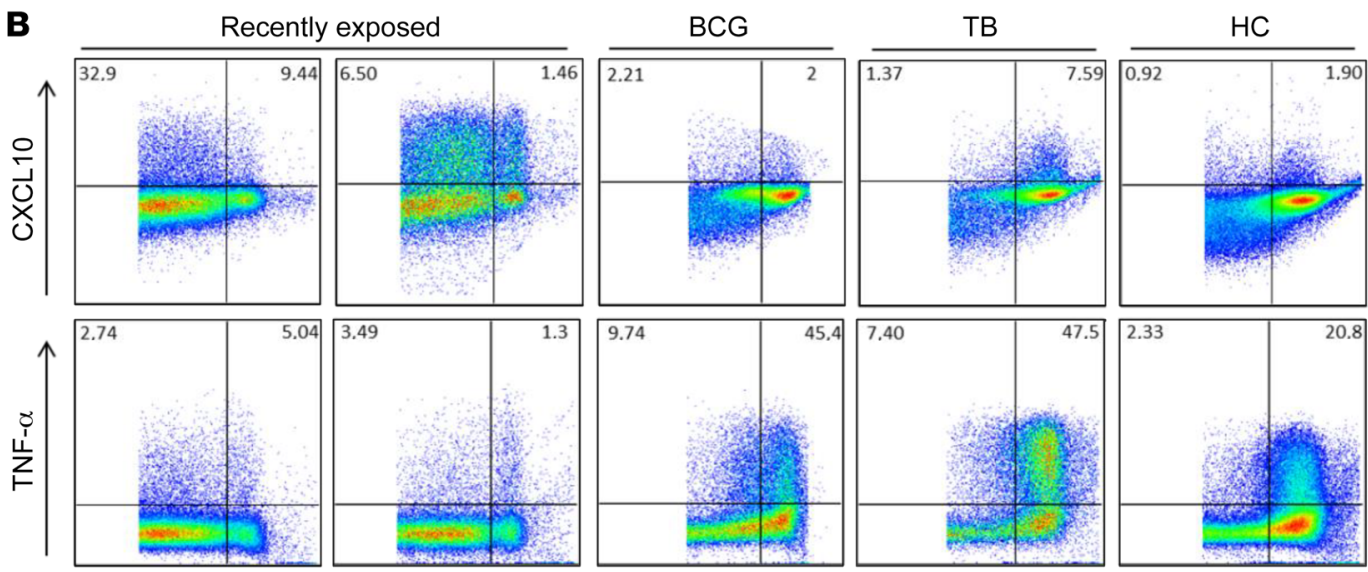

CD14

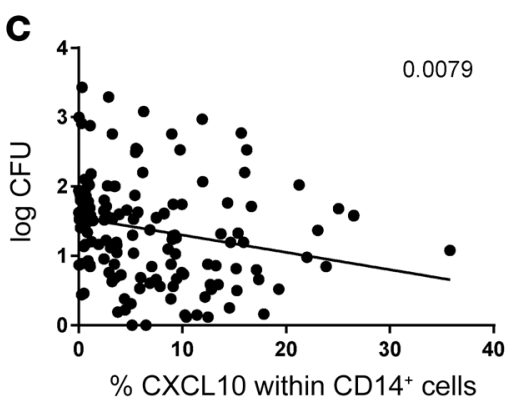

$\mathbf{F}$

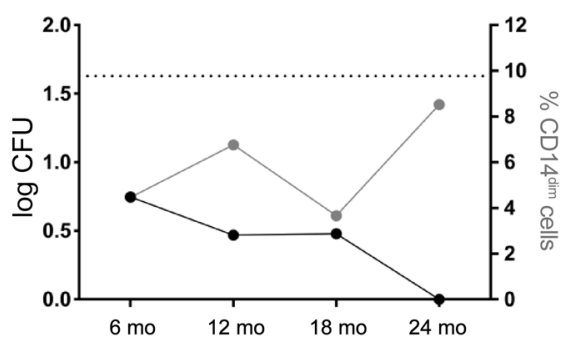

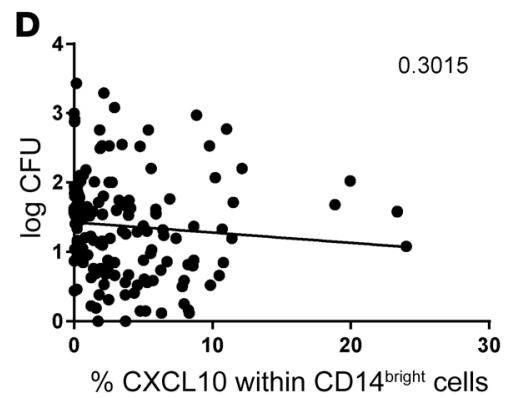

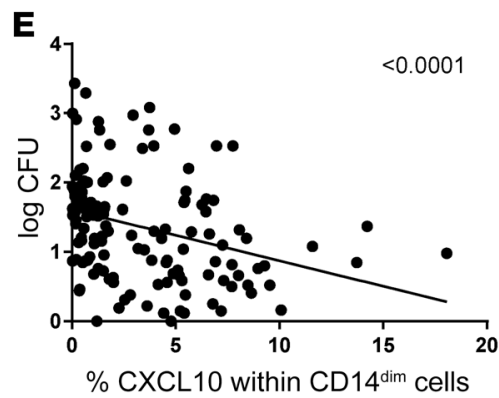

G
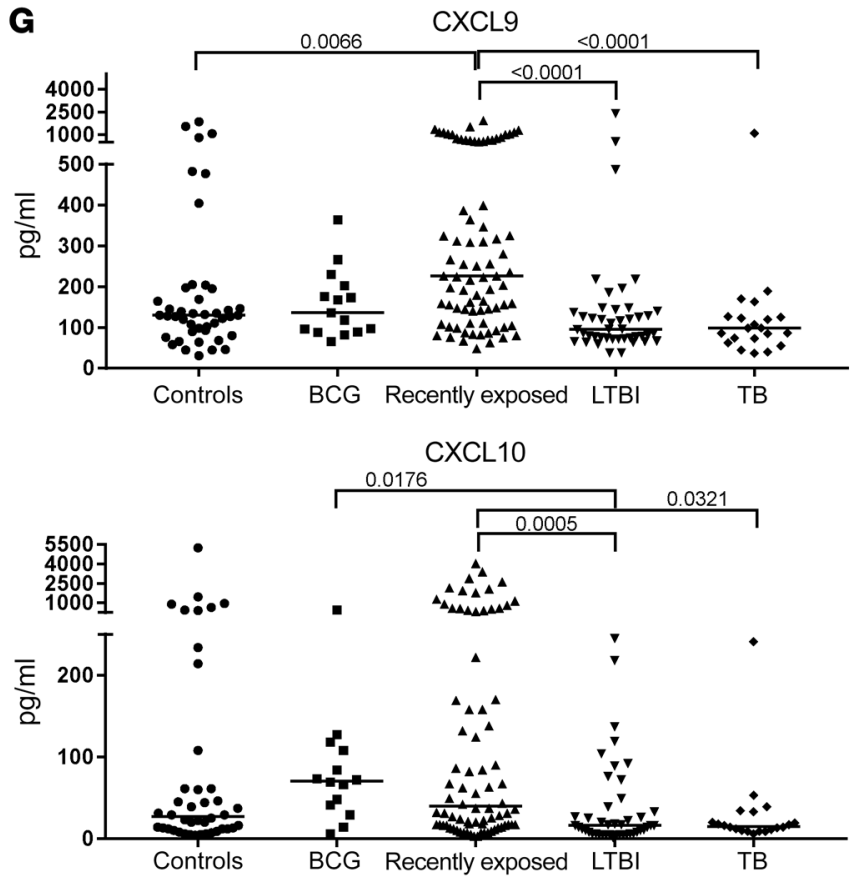
Figure 4. Nonclassical monocytes produce CXCL10 and are involved in mycobacterial growth control. PBMCs were infected with live BCC and rotated for 4 days, and samples were incubated in MCIT tubes in the BACTEC machine. Antibody marker staining for FACS analysis was performed after 16 hours in the absence of specific stimulation in the presence of BFA. Associations were determined using linear regression modeling, and $P$ less than 0.05 was considered significant. (A) Concatenated gate of CD14 dim and CD14 bright cells for monocyte subset markers revealed a nonclassical monocyte expression pattern of the CD14 ${ }^{\mathrm{dim}}$ population consisting of low CD163, low CCR2, high CX3CR1, and high CCR5 expression. (B) CXCL10 (top row) and TNF- $\alpha$ (bottom row) production by monocytes in recently exposed individuals, a BCG-vaccinated donor, a TB patient, and a healthy control (HC) donor. (C) The percentage of total monocyte-derived CXCL10 was positively correlated with the capacity to control mycobacterial outgrowth $(n=144)$. (D) The percentage of CD14 ${ }^{\text {bright }}$ cells producing CXCL10 did not correlate with mycobacterial growth control $(n=144)$. (E) The percentage of CD14 dim cells producing CXCL10 correlated strongly with mycobacterial growth control $(n=144)$. (F) Longitudinal follow-up of individuals from the supermarket contact investigation revealed increasing levels of growth control, which coincided with an increased percentage of CXCL10-producing CD14 dim cells (line indicates mean of 8 samples). (C) Supernatants of 4-day MGIA cocultures were tested for cytokine and chemokine production using a multiplex bead array. Shown are CXCL9 and CXCL10 production and CXCL9 production, expressed as picograms per milliliter, over the clinical groups. Groups were compared using the Kruskal-Wallis test. Controls, $n=43$; BCG, $n=15$; exposed, $n=79$; LTBI, $n=44 ;$ TB, $n=21$.

In contrast, however, analysis of different memory $\mathrm{T}$ cell populations revealed a strong association $(P<0.0001)$ of $\mathrm{CD}^{+}{ }^{+}$central memory $\mathrm{T}$ cells with the capacity to control BCG outgrowth (Figure 2E; gating strategies are given in Supplemental Figure 7C). Both $\mathrm{CD} 4^{+}$and $\mathrm{CD} 8^{+}$effector cell frequencies were inversely related with MGIA control (Figure 2F and all data in Supplemental Figure $4, \mathrm{~A}$ and $\mathrm{B})$.

In addition to cells involved in adaptive immunity, we also assessed monocytes, being the primary targets of BCG infection. The percentage of $\mathrm{CD} 14^{+}$monocytes correlated significantly with the capacity to control BCG outgrowth in the MGIA $(P=0.0006)$ (Figure 3A). An even stronger correlation with MGIA activity was seen when we expressed monocyte/lymphocyte (ML) ratios, which were obtained by division of the percentage of $\mathrm{CD} 14^{+}$cells by that of $\mathrm{CD}^{+}$cells $(P<0.0001)$ (Figure 3B). When analyzing these $\mathrm{CD} 14$ data we noticed that particular samples had a very abundant CD14 dim population (Figure 3C). Therefore, we analyzed CD14 ${ }^{\mathrm{dim}}$ and $\mathrm{CD} 14^{\text {bright }}$ populations separately and found that the $\mathrm{CD} 14^{\mathrm{dim}}$ population was most abundantly present in individuals recently exposed to Mtb (Figure 3D). Interestingly, not the frequency of the CD14 $4^{\text {bright }}$ (Figure $3 \mathrm{E}$ ) but that of CD14 $4^{\text {dim }}$ cells $(P<0.0001)$ (Figure $3 \mathrm{~F})$ correlated with the capacity to control $\mathrm{BCG}$ outgrowth in the MGIA.

Thus we have identified a CD14 ${ }^{\mathrm{dim}}$ monocyte subset that is increased upon recent exposure to Mtb, and correlates with the capacity to control BCG outgrowth.

Nonclassical monocytes are associated with MGIA control and production of CXCL1O. CD14 ${ }^{\text {dim }}$ monocytes are nonclassical monocytes that are further characterized by the expression of particular chemokine receptors. Indeed, $\mathrm{CD} 14^{\text {bright }}$ cells expressed increased levels of CD163, CCR2, and CX3CR1, but CD14 dim cells expressed higher levels of CCR5, consistent with a nonclassical monocyte subset phenotype (Figure 4A). Further work revealed that a large proportion of the $\mathrm{CD} 14^{\mathrm{dim}}$ population produced the chemokine CXCL10. In contrast, TNF- $\alpha$ was generally produced by CD14 $4^{\text {bright }}$ cells (Figure $4 \mathrm{~B}$ ). Most importantly, the proportion of CXCL10-producing monocytes was associated with the capacity to control BCG outgrowth (Figure 4C). The capacity to control BCG outgrowth was associated not with CXCL10-producing CD14 ${ }^{\text {bright }}$ cells (Figure 4D), but with CXCL10-producing CD14 dim cells (Figure 4E). The proportion of total $\mathrm{CD} 14^{+}, \mathrm{CD} 14^{\text {bright }}$, or CD14 ${ }^{\text {dim }}$ cells producing TNF- $\alpha$ did not correlate with the capacity to reduce BCG outgrowth (Supplemental Figure 5, A-C).

Interestingly, follow-up of individuals exposed in the supermarket outbreak allowed longitudinal assessment of the capacity to control BCG outgrowth. The capacity to control BCG outgrowth persisted during the first 2 years after exposure, and time points with the strongest reduction in BCG outgrowth contained the highest frequency of CD14 $4^{\mathrm{dim}}$ monocytes (Figure $4 \mathrm{~F}$ ). Longitudinal follow-up was available only for the supermarket study and for the Dutch cohort of LTBI individuals. In the supermarket contact investigation with recent Mtb exposure, control in the MGIA was maintained throughout the 2-year follow-up. Although strong control was achieved, this did not result in sterile eradication of the BCG in the MGIA assay (Supplemental Figure 6A). In the more remote LTBI cohort, however, no control was observed during follow-up (Supplemental Figure 6B). In agreement with the above results, the supermarket group had increased ML ratios, increased frequencies of $\mathrm{CD} 14^{\mathrm{dim}}$ cells, and increased frequencies of CXCL10-producing CD14 $4^{\mathrm{dim}}$ cells, whereas none of these were observed during follow-up of the LTBI cohort (Supplemental Figure 6, C-H).

Thus, our functional and immunological analyses identify strong correlations between the capacity to control BCG outgrowth in the MGIA and the presence of a nonclassical monocyte subset characterized by decreased expression of CD14, that produced CXCL10.

Mechanistic involvement of CXCL1O/CXCR3 signaling requires the presence of $T$ cells. Since the frequency of CXCL10-producing CD14 ${ }^{\mathrm{dim}}$ monocytes was strongly associated with MGIA control, we next investigated the concentration of CXCL10 and its closely related family member CXCL9 in the supernatants of the 4-day MGIA assay. CXCL9 and CXCL10 were present at the highest levels in donors recently exposed to $\mathrm{TB}$, although concentrations varied between individuals (Figure 4G). The third CXCR3-binding family member, CXCL11, could not be detected in the same MGIA supernatants. CXCL9 and CXCL10 bind to CXCR3 as their cognate receptor. NBI-74330, a chemical CXCR3 receptor antagonist, was added to the MGIA assay during the 4 days of incubation. Controls with the CXCR3 antagonist and BCG bacteria only, in the absence of PBMCs, revealed no difference in BCG outgrowth kinetics, excluding direct effects of the inhibitor on bacterial growth. Addition of the inhibitor to PBMCs from samples that controlled BCG outgrowth abrogated the reduction in BCG CFU, indicating that the CXCR3/CXCL10 axis is functionally involved in controlling BCG outgrowth (Figure 5A; raw data given in Supplemental Table 1). Interestingly, PBMC samples that lacked BCG outgrowth control showed increased capacity to control BCG outgrowth upon addition of the CXCR3 antagonist NBI-74330, to a small but significant extent (Figure 5B). Analysis of the supernatants of these cocul- 
A
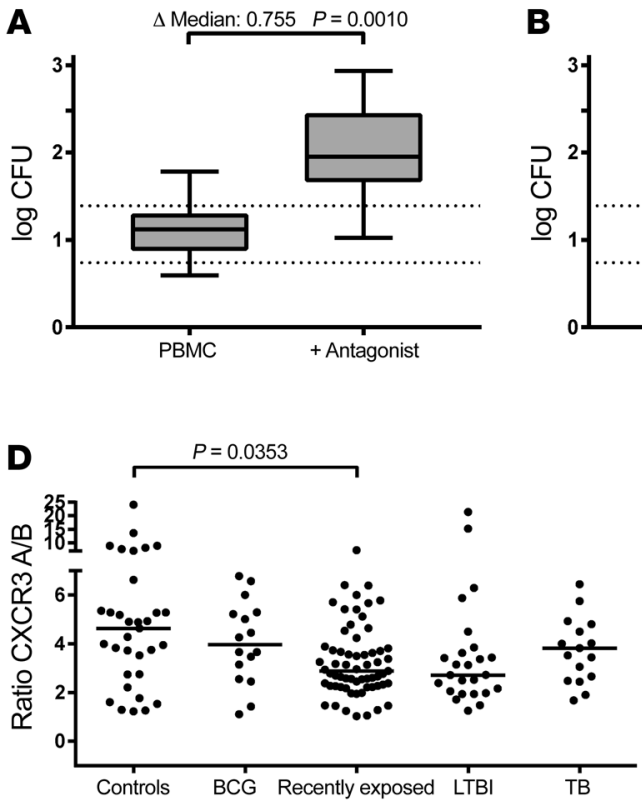
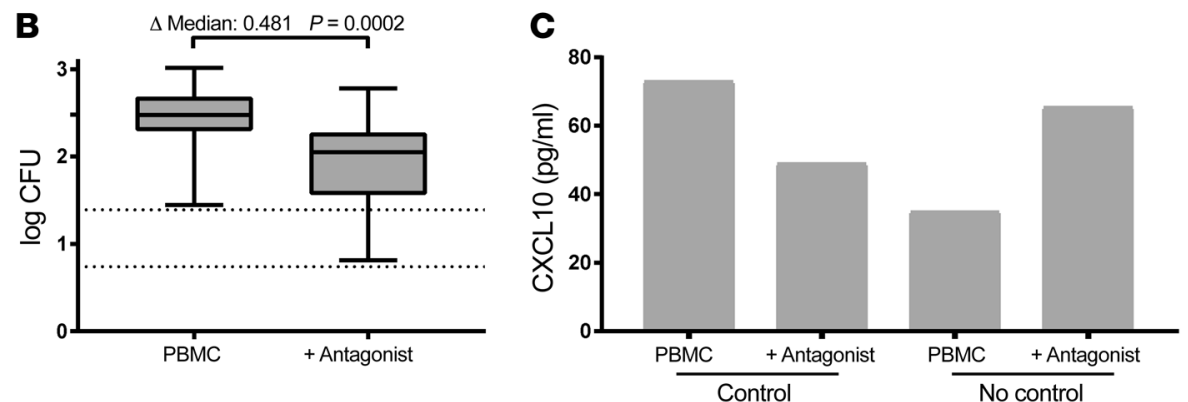

$\mathbf{E}$

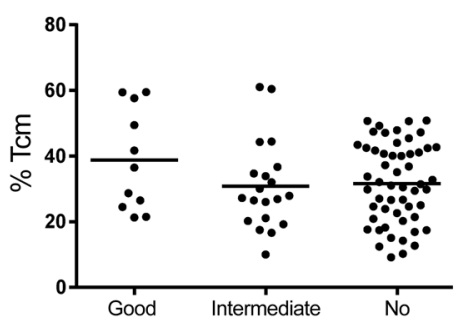

$\mathbf{F}$

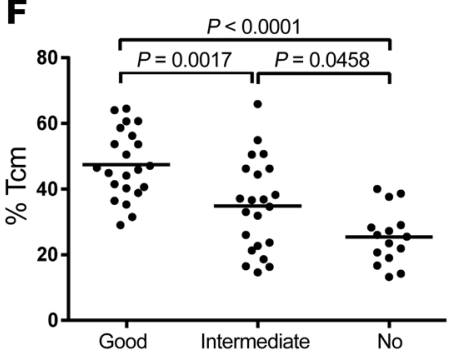

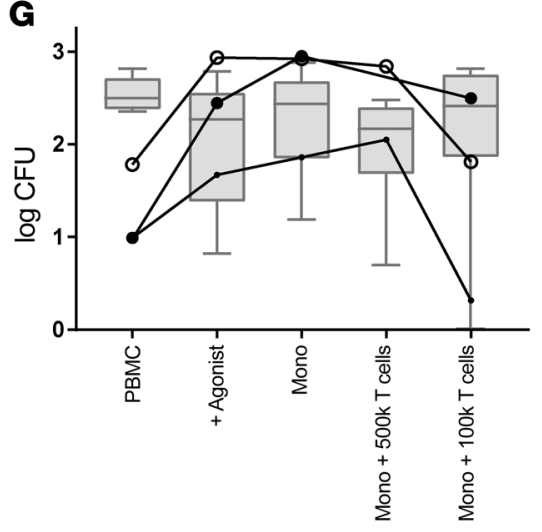

Figure 5. CXCR3 is key in determining mycobacterial outgrowth control. PBMCs were infected with live BCC and rotated for 4 days, and samples were incubated in MGIT tubes in the BACTEC machine. (A) A CXCR3 receptor antagonist (NBI-74430) was added daily during the MCIA incubation period to inhibit activity of CXCL10; this reverted the capacity to control mycobacterial outgrowth. Data represent 11 donors with MCIA control run in 2 independent experiments; differences were assessed using the Wilcoxon matched-pairs signed-rank test. (B) Similar to $\mathbf{A}$, addition of the CXCR3 receptor antagonist during the MGIA assay. Data represent 27 donors without MGIA control run in 3 independent experiments; differences were assessed using the Wilcoxon matched-pairs signed-rank test. (C) CXCL10 production was measured in supernatants of the MCIA assay at day 4 of coculture in samples with MGIA control or no MGIA control, in the absence or presence of NBI-74430, the CXCR3 antagonist. Data are expressed as median. (D) CXCR3 splice variants were determined by real-time quantitative reverse transcriptase PCR. $\triangle$ Ct was calculated for both CXCR3A and CXCR3B using the in-well GAPDH control. Subsequently, the ratio of CXCR3A over CXCR3B was calculated and plotted. Data were compared using the Kruskal-Wallis test. Controls, $n=33$; BCG, $n=16$; exposed, $n=$ 63; LTBI, $n=26$; TB, $n=16$. (E) The percentage of Tcm cells was plotted for donors with a low frequency of CD14 dim monocytes producing CXCL10 ( $<3 \%)$. Good, $n=11$; intermediate, $n=20 ;$ no, $n=54$. (F) The percentage of Tcm cells plotted for donors with a high frequency of CD14 dim monocytes producing CXCL10 ( $>3 \%$ ). Groups were compared using the Kruskal-Wallis test. Good, $n=22$; intermediate, $n=22 ;$ no, $n=15$. (C) MGIA assay with PBMCs and isolated fractions of monocytes with or without $T$ cells. Cells were separated and combined in various ratios before infection with live BCC and 4-day coculture. Boxes and whiskers indicate donors that lack MGIA control $(n=8)$; lines indicate 3 individual donors with MGIA control. tures revealed a similar pattern in the production of CXCL10 at day 4 of the cultures, suggesting a feedback loop between chemokine production and receptor function (Figure $5 \mathrm{C}$ ).

These opposing effects of the CXCR3 antagonist in donors that controlled versus those that did not control BCG outgrowth prompted us to assess expression of CXCR3 receptor alternative splice variants. Interestingly, although these splice variants differ only in the N-terminal part of this 7-transmembrane G proteincoupled receptor, their intracellular effector coupling is different $(40,41)$ : CXCR3A is an immune-activating receptor and has the highest binding affinity for CXCL9, CXCL10, and CXCL11, whereas CXCR3B exerts a more inhibitory role and can also bind CXCL4 (42). The regulation of expression of each of the splice variants has not been resolved yet, but epigenetic modifications may play a role.

RNA samples were isolated from most PBMC samples (depending on cell yields) directly after thawing, without any stimulation, and stored. TaqMan quantitative reverse transcrip- tase PCR was performed for both splice variants on all samples that were available. Recently exposed individuals had a decreased ratio of CXCR3A over CXCR3B, as a result of both increased CXCR3B and reduced CXCR3A expression (Figure 5D). LTBI donors showed a similar trend toward a decreased ratio, but this did not reach statistical significance.

The CXCR3 receptor is most abundantly expressed on T cells, although it can also be expressed on monocytes, NK cells, and B cells. Since all MGIA data were obtained from total PBMCs, we wondered whether monocytes alone would be sufficient or whether the presence of $\mathrm{T}$ cells was required. First, data were compared for the percentage of central memory $\mathrm{T}$ cells (Tcm cells), since these cells typically express CXCR3 and may be responsible for the effector function (43). Since the presence of CXCL10-producing CD14 ${ }^{\mathrm{dim}}$ monocytes seems a first prerequisite for BCG control, groups were subdivided based on the percentage of CXCL10-positive cells as determined in Figure 4E, using a cutoff of $3 \%$. Indeed, donors with good control of BCG outgrowth had 
higher frequencies of Tcm cells, suggesting that $\mathrm{T}$ cells and/or $\mathrm{T}$ cell-expressed CXCR3 is involved in control of BCG outgrowth (Figure 5, E and F). Second, we performed an additional series of MGIA experiments, in which we included not only total PBMCs, but also isolated monocytes and monocytes supplemented with purified autologous T cells. PBMCs from BCG controllers again inhibited outgrowth of BCG, which could be reversed with the CXCR3 antagonist as shown above (Figure 5G). However, isolated monocytes did not reduce the bacterial burden unless $\mathrm{T}$ cells were added (Figure $5 \mathrm{G}$ ). Thus, both monocytes and $\mathrm{T}$ cells are required to control BCG outgrowth.

Trained immunity. Since we observed control of BCG outgrowth mostly in donors that were recently exposed to Mtb and since control of BCG outgrowth was associated with a particular subset of monocytes, we evaluated whether trained innate immunity was responsible for the observed bacterial control. Trained immunity has previously been described in studies following BCG vaccination and in vitro restimulation with unrelated pathogen-derived pattern recognition receptor agonists $(28,29,44)$. Cytokines that thus far have been reported as primary indicators of trained immunity are IL- $1 \beta$, IL- 6 , and TNF- $\alpha$. Samples from the MGIA assays of the above tested clinical groups were therefore analyzed for the production of these cytokines. Confirming our hypothesis, all 3 were found at increased levels in recently Mtb-exposed individuals (Figure 6, A-C).

Next, in order to confirm that our above-defined CXCR3-binding chemokines are indeed associated with trained immunity, we assessed their concentrations in an independent cohort of recently BCG-vaccinated individuals from whom supernatants with classical trained immunity were obtained 4 weeks after vaccination. PBMCs isolated from volunteers vaccinated with placebo or BCG were restimulated for 24 hours in vitro with heat-killed Candida albicans, Staphylococcus aureus, Mtb, or lipopolysaccharide. Although CXCL9 was detected in these BCG-trained samples (Figure 6D), CXCL10 was much more strongly upregulated (Figure 6E). CXCL11 was also found to be increased in the classical trained immunity samples (Figure 6F). These data show that CXCL9, CXCL10, and CXCL11 may be newly identified markers of trained immunity.

Additional cytokine and chemokine data were collected using a 40-plex bead array. We also analyzed these cytokines and chemokines for their induction both in classically trained samples and in our recently exposed individuals. Intriguingly, along with CXCL9, CXCL10, and CXCL11, also IL-4, CCL8 (MCP2), CCL11 (eotaxin), CCL17 (TARC), CCL19 (MIP3ß), CCL23 (MIP3), CCL25 (TECK), CCL26 (MIP4 $\alpha$ ), CCL27 (C-TACK), and CXCL13 (BLC) were identified as potential markers of trained immunity, deserving further research (Figure 6G).

Hence, we identified new markers of trained immunity and a series of candidate markers that deserve further investigation for their possible contribution to trained immunity effector mechanisms. The CXCR3 axis, involving both CXCL9 and CXCL10 as ligands, has a functional role in mediating mycobacterial outgrowth control in the context of trained immunity. Monocyte frequency, and in particular the CD14 ${ }^{\mathrm{dim}}$ nonclassical monocyte population that produced CXCL10, may serve as surrogate markers for BCG outgrowth control. However, it remains important to vali- date these findings in additional, independent cohorts, preferably composed of recently exposed individuals. In addition, validation of these findings in human or nonhuman primate studies with novel vaccine candidates that induce protection, when available, would be extremely valuable. Detailed analysis of samples with strong control of bacterial outgrowth may contribute to the identification of novel correlates of protection.

\section{Discussion}

The present study reports that mycobacterial growth inhibition can be measured in humans using a refined and robust MGIA assay. Contrary to our expectation, improved mycobacterial growth control was not associated with (Th1) cytokine-producing (or polyfunctional) $\mathrm{T}$ cells, but rather was associated with the presence and activity of a nonclassical CD14 ${ }^{\text {dim }}$ monocyte subset, but Tcm cells were required for growth control. Individuals with recent, but not remote, exposure to Mtb had the strongest capacity to control BCG outgrowth, suggesting that a temporary change in effector immunity occurred that mediated growth control. Indeed, nonclassical monocytes producing CXCL10 were found to be involved in BCG growth control through the CXCR3 receptor. Expression of CXCR3 splice variants, with immune-activating or -inhibiting functions, was altered upon recent Mtb exposure. Moreover, both CXCR3 ligands, CXCL9 and CXCL10, were also detected in samples of recently BCG-vaccinated individuals in which trained immunity was induced, suggesting that trained innate immunity was the mechanism behind control of BCG outgrowth in the MGIA following recent exposure to Mtb.

Mycobacterial growth control has been considered an important new readout of the capacity of the immune system to control BCG or Mtb, particularly because these assays are functional and do not require a specific a priori hypothesis regarding possible mechanisms involved. Since TB vaccines are designed to induce protective $\mathrm{T}$ cell immunity, the responsible immune effector cells in the MGIA assay have also been presumed to be T cell subsets. Recently, Smith et al. suggested an association between MGIA control capacity and the frequency of multifunctional $\mathrm{CD} 4^{+} \mathrm{T}$ cells, studying a small cohort of BCG-vaccinated infants (25). However, these data could not be replicated in our much larger cohort of individuals from different settings. In our view, the frequencies of multifunctional T cells observed in both Smith's and our study were extremely low; moreover, the negative control populations that lack both multifunctional T cells and MGIA control skewed the trend lines strongly. Therefore, we conclude that multifunctional $\mathrm{T}$ cells are not associated with the capacity of human PBMCs to control BCG growth, at least in our larger cohorts, but likely also in others. Previous studies in mouse models of MGIA identified a role for IFN- $\gamma$ in the control of BCG outgrowth (11). IFN- $\gamma$-deficient mice had reduced growth control, but these effects were most clear in conditions in which low numbers of splenocytes and relatively high BCG inocula were used (11).

Also in line with this observation is our finding that a considerable proportion of individuals who lack cellular immune responses against Mtb, in TST and/or by IFN- $\gamma$ production in QFN, controlled BCG outgrowth quite well. Similarly, two-thirds of recently exposed individuals from the soccer club contact investigation did not convert their TST. All TST-negative individuals were tested 
A

IL-1 $\beta$

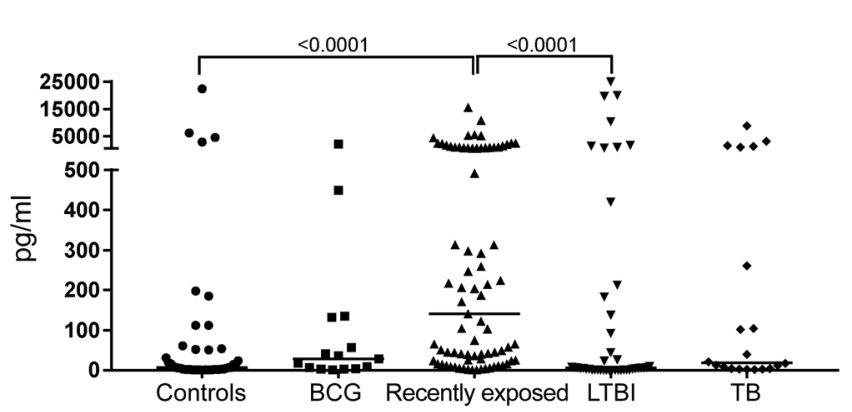

C

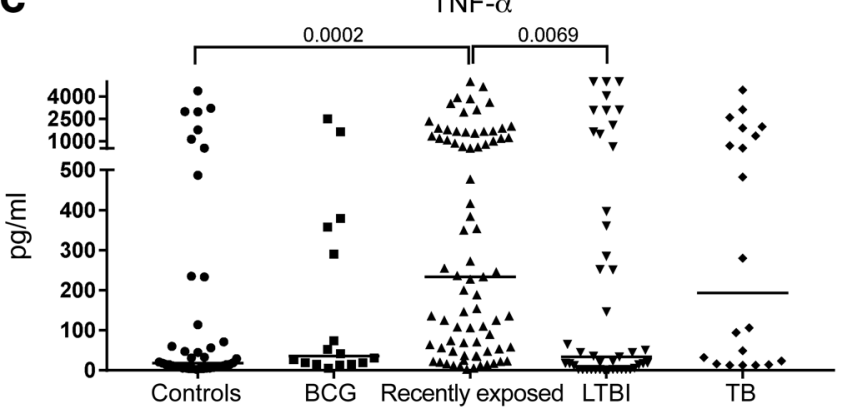

B

IL-6

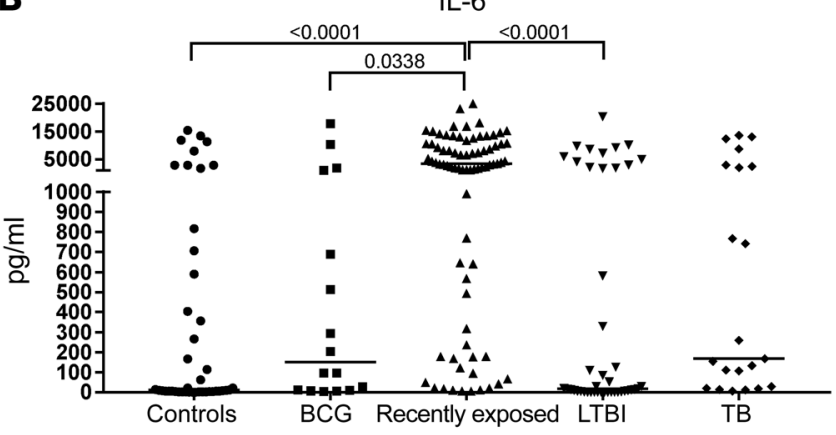

D

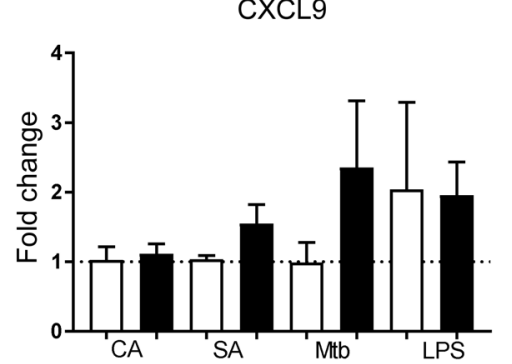

E

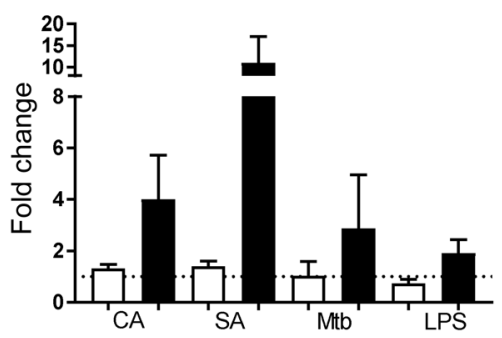

$\mathbf{F}$

CXCL11

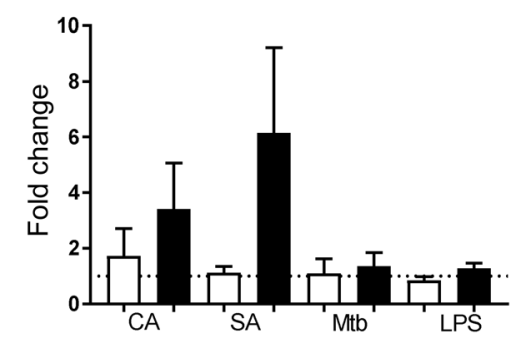

G Fold change

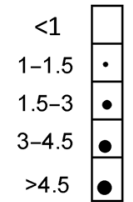

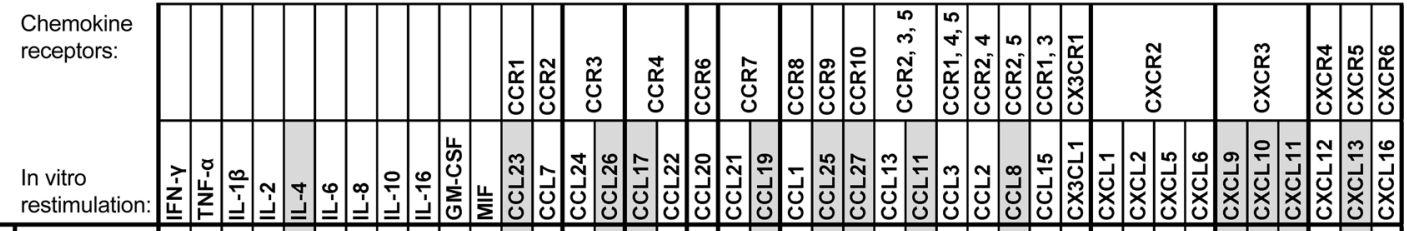

Trained

Trained
immunity by

BCG

restimulation:

vaccination

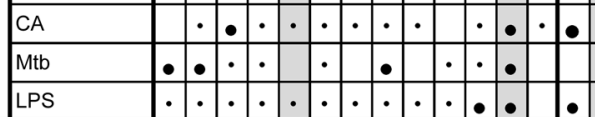

Subcohort:

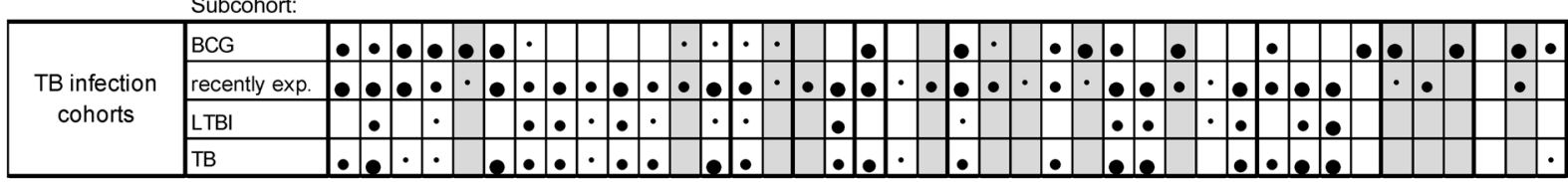


Figure 6. CXCL10 production is a marker of trained immunity. Supernatants from the 4-day coculture with BCG were collected and cytokine/chemokine production determined using a multiplex bead array. In addition, samples were collected from in vivo, BCG-vaccinated individuals and stimulated ex vivo (restimulation with microbiological agents). Groups were compared using the Kruskal-Wallis test (A-C). (A) IL-1 $\beta$ production (as classical trained immunity marker) was assessed in 4-day culture supernatant. Controls, $n=43$; BCG, $n=15$; exposed, $n=79$; LTBI, $n=40$; TB, $n=20$. (B) IL-6 production (as classical trained immunity marker) was assessed in 4-day culture supernatant. Controls, $n=43$; BCC, $n=16$; exposed, $n=79$; LTBI, $n=44$; TB, $n=21$. (C) TNF- $\alpha$ production (as classical trained immunity marker) was assessed in 4-day culture supernatant. Controls, $n=42$; BCG, $n=16$; exposed, $n=78$; LTBI, $n=45$; TB, $n=20$. (D) CXCL9 (as new trained immunity marker) was measured in supernatants of ex vivo-stimulated PBMCs. Black bars indicate BCG-vaccinated donors $(n=10)$; open bars indicate placebo-vaccinated donors $(n=5)$. Results are expressed as fold change of 4 weeks post-vaccination over the prevaccination time point. CA, Candida albicans; SA, Staphylococcus aureus. (E) CXCL10 (as new trained immunity marker) was measured and expressed similarly as in $\mathbf{D}$. (F) CXCL11 (as new trained immunity marker) was measured and expressed similarly as in $\mathbf{D}$. (C) Summary of all cytokine and chemokine data collected in the multiplex bead array analysis using supernatants from individuals with trained immunity following BCG vaccination (top part; vaccinated donors only) and samples from individuals from TB infection cohorts from the current study (bottom parts). Dot sizes indicate the fold change compared with prevaccination for the trained cohort and fold change versus the control population in our clinical TB infection groups. Gray-shaded cytokines and chemokines are potentially associated with trained immunity.

by TST a second time 6 weeks after initial TST reading, but many remained negative. When the capacity to control BCG outgrowth in the MGIA was compared in TST-positive $(\geq 10 \mathrm{~mm})$ versus TST-negative recently exposed individuals, no differences were observed; both subgroups controlled BCG outgrowth equally well. We therefore hypothesized that these individuals may efficiently have controlled bacterial growth through innate immunity, which could imply that induction of adaptive immunity was not needed and the TST therefore remained negative.

The ML ratio was very strongly associated with the capacity to control BCG outgrowth. Previous studies have also identified ML ratio as an important factor associated with the capacity to control in MGIA; however, a whole-blood version of the MGIA was used in that study, precluding a direct comparison with our data (24). Intriguingly, transcriptomic analysis of blood monocytes from those individuals identified IFN- $\gamma$ among the top 5 upstream regulators, together with type I IFNs associated with early responses against intracellular infections (24). IFNs can have direct effects, but also augment effector responses through induction of several downstream inflammatory mediators such as IFN-inducible protein 10 (IP-10) or CXCL10. Thus, early activation of these transcriptomic pathways in monocytes may also result in activation of CXCL10 and some of its family members. Gene expression studies in BCG-vaccinated mice already had identified CXCL9 as one of the most differentially expressed genes (12). Stimulation with BCG resulted in strong transcriptional upregulation of CXCL9 as well as CXCL10, in addition to TNF- $\alpha$, IL-1 $\beta$, and IL-6 (12), fitting perfectly with our trained immunity hypothesis. Also, in studies with the novel vaccine candidate MVA-Ag85A, CXCL10 was the top differentially transcribed gene at day 1 after vaccination both in South African infants and in UK adults (45). Vaccinated infants at day 28 also expressed more CXCL9 and CXCL10 compared with their prevaccination sample (45). Monocyte signatures were most abundantly expressed and correlated with increased $\mathrm{T}$ cell responses to vaccination (45). These findings indicate that early activation of innate immune cells, with production of their respective cytokines, is critical for guiding the adaptive immune system.

Unexpectedly, nonclassical CD14 ${ }^{\mathrm{dim}}$ monocytes were found to correlate strongly with the capacity to control BCG outgrowth. This cell population was abundantly present in individuals with recent TB exposure and strong MGIA growth reduction. Nonclassical monocytes have been associated with a predominantly scavenging and sensing function within the immune system, in particular in the case of intracellular viral infections (46). These cells may also be involved in local tissue surveillance and have been linked to autoimmune diseases (46). Nonclassical monocytes are efficient recognizers of extracellular TLR ligands, but also of immune complexes. TLR triggering did not induce ROS or cytokine production by CD14 ${ }^{\mathrm{dim}}$ cells, but triggered specific production of TNF- $\alpha$, IL-1 $\beta$, and CCL3, e.g., by virus-induced TLR7/8 triggering (47). TLR7/8 triggering also induced CXCL10 production by these cells, perhaps via a slightly different signaling pathway, since MYD88 was not essential for CXCL10 production, whereas it is required for TNF- $\alpha$ and IL-1 $\beta$ production (47). In addition, besides their ability to release multiple cytokines, CD14 ${ }^{\mathrm{dim}}$ cells are also highly motile, in agreement with their patrolling function, but are relatively weak phagocytes (47).

Intracellular analysis of several cytokines and chemokines revealed that the $\mathrm{CD} 14^{\mathrm{dim}}$ monocyte population produced the CXCR3 ligand CXCL10, and CXCL10 production correlated strongly with BCG growth reduction. Indeed, CXCR3 receptor blockade using a chemical antagonist confirmed mechanistic involvement of the CXCR3 signaling pathway in BCG growth reduction. CXCR3 can bind not only CXCL10, but also CXCL9 and CXCL11. These ligands were not present in our intracellular staining panel, but CXCL9, not CXCL11, was abundantly detected in supernatants. It remains to be seen whether a single or rather a combination of CXCR3 ligands is responsible for mediating BCG growth reduction. The effect of these chemokines may further depend on the balance in expression of splice variants of CXCR3, since in recently $\mathrm{Mtb}$-exposed individuals a decreased ratio of CXCR3A over CXCR3B was observed. Although monocyte alterations were most strongly associated with changes in control of BCG outgrowth, the presence of T cells was found to be indispensable to mediate ultimate BCG growth control by monocytes. In any event, we think this is the first molecular identification of a host effector molecule responsible for mycobacterial growth inhibition, besides granulysin $(48,49)$.

Since our data suggested that the cellular ability to control mycobacterial growth was associated with recent exposure to Mtb, was transient, and was linked to the (nonclassical) monocyte phenotype and (CXCR3 signaling-related) function, we hypothesized that this ability may have been acquired as a result of trained innate immunity by epigenetic changes in the myeloid cell lineage $(28,50)$. "Trained immunity" refers to the altered functional state of innate immune cells that persists after elimination of the initial stimulus, and allows an increased response to restimulation of the cells through both the same and different pattern recognition receptors $(50,51)$. 
In humans, we (M.G. Netea and colleagues) have shown that BCG vaccination induces trained immunity $(28,50)$. Indeed, cytokines previously associated with trained immunity such as TNF- $\alpha$, IL- $1 \beta$, and IL- 6 were also increased in the current study in individuals with recent exposure to $\mathrm{Mtb}$, suggesting trained immunity in these samples. Importantly, samples from previous trained immunity studies were shown in the current study to contain large amounts of CXCL10, and also CXCL9 and CXCL11, thus bridging these previous trained immunity studies now with our current TB cohort observations, and newly identifying CXCL9 and CXCL10 as markers of trained immunity. Future studies on the epigenetic alterations of these genes will be required to fully establish these chemokines as novel trained immunity markers.

Trained immunity-associated cytokines were detected in the majority of individuals with recent exposure to Mtb, but only in a limited number of individuals with more remote LTBI or TB disease. This suggests that Mtb infection-induced training of monocytic cell lineages is a temporary phenomenon during the first period after infection. This is in line with our earlier observation that BCG vaccination exerts strong trained immunity effects for several months, but these effects diminish 1 year after vaccination (29). It is difficult to estimate the duration of persisting trained immunity after recent exposure, in particular in Mtb infection, since in many cases the exact timing of infection is unknown. However, the contact investigation in the supermarket allowed identification of the presumed infectious period and included longitudinal follow-up (37). Somewhat to our surprise, most contacts from that outbreak maintained the capacity to control BCG in the MGIA during the 2 years of follow-up from initial exposure. The lifespan of circulating monocytes is estimated to be in the magnitude of days, implying that training must occur at the monocytic progenitor level to allow longer-lasting training effects. Mycobacteria have been found in human and mouse stem cells (52) in the bone marrow, and it is possible that this niche could be involved in training innate immune cells. Future studies in animals would be needed to demonstrate a causal link between functional reprogramming of myeloid cell progenitors and trained immunity in TB.

BCG-induced trained innate immunity may underlie some well-known observations, such as that infants vaccinated with BCG are protected from secondary infections against unrelated pathogens (53). Epidemiological studies suggested that these effects can last for several months until up to possibly multiple years (54). Circulating monocytes with trained immunity functional phenotypes have been detected at 3 and 12 months following BCG vaccination (29). Epidemiological evidence has furthermore indicated that prior LTBI strongly protects against development of active TB upon reinfection (27). Moreover, BCG vaccination has the highest efficacy in the absence of prior sensitization or preexisting infection (55). In analogy with these epidemiological findings, experimental vaccination studies revealed that MGIA growth reduction is induced by priming BCG vaccination but not upon secondary BCG vaccination (9). The mechanisms behind this protective efficacy in primary infection/vaccination have not been resolved, but may be explained by continuous innate immune training, which is capable of limiting unrelated as well as related bacterial growth upon reinfection. Obviously, while this new hypothesis will need to be corroborated further in suitable animal models such a nonhuman primate, collectively these observations point to an important role for innate and trained immunity in protective immunity against mycobacterial infections. These initial events are likely also important in guiding and steering the adaptive response, which is key to optimal protection as well. How exactly these different phenomena are causally, mechanistically, and temporally related remain topics for further study.

Recently, a mouse MGIA study was published that showed control of Mtb Erdman outgrowth in vaccinated mice (14). However, mice vaccinated only with the CAF01 adjuvant also showed such control, which was interpreted as an artifact. However, this observation could also fit with the induction of trained immunity. Moreover, the authors were unable to associate growth control with the number of cytokine-producing vaccine-specific $\mathrm{T}$ cells and found that there was no persistence of growth reduction at 29 weeks after vaccination (14). These data in fact fit well with the actual induction of temporary trained innate immunity as we report here.

Trained immunity has been described for monocytes. However, other circulating cells may also be instructed as a result of pathogen-induced training. MGIA control was associated not only with monocytic cell populations but also with $\mathrm{B}$ cells and $\mathrm{CD} 4^{+}$ central memory cells. In contrast, numbers of total $\mathrm{CD}^{+} \mathrm{T}$ cells as well as $\mathrm{CD}^{+}$and $\mathrm{CD} 8^{+}$effector cells inversely correlated with mycobacterial growth control.CXCR3 expression is most abundant on CD4 memory T cells, and T cells were required to mediate BCG growth control, as isolated monocytes were unable to control BCG outgrowth. These data suggest an interplay between T cells and monocytes in the control of BCG outgrowth through the CXCL10/CXCR3 axis. It has previously been reported that the $\mathrm{CXCR}^{+} \mathrm{CCR}^{+}$memory $\mathrm{T}$ cell population contained the vast majority of anti-Mtb reactive Th1 cells, and these rather unusual Th1 cells have been coined Th1* $(43,56)$. Thus not only innate cells but also CXCR3-expressing Mtb-specific $\mathrm{T}$ cells may be directed by CXCL10 produced by trained monocytes to mediate Mtbspecific immunity. An additional cell population in which trained immunity has been shown to be induced by BCG vaccination are NK cells (57). The importance of trained NK cells for protection has also been suggested by a recent clinical trial in which correlates of protection after BCG vaccination were reported to be not $\mathrm{CD}^{+} \mathrm{T}$ cells, but IFN- $\gamma$-producing NK cells (58).

Although MGIA has been developed in principle as an in vitro measure of vaccine-induced protection, our data now show that growth control in the MGIA was the result of trained innate immunity, rather than of effector $\mathrm{T}$ cell induction. However, $\mathrm{T}$ cells appear to be involved in execution of growth control. To date, BCG is the only vaccine that induced partial protection against TB in humans, and it is well known to induce trained innate immunity. However, multiple new vaccine candidates are currently in clinical testing, and it is hard to speculate whether these vaccines induce protection in humans and whether these use (different) effector mechanisms. Therefore, we recommend future studies that analyze mechanisms of protection against mycobacteria to take into account not only effector $\mathrm{T}$ cell induction but also trained innate immunity. 
Originally, trained immunity was demonstrated measuring the production of IL- 6 , TNF- $\alpha$, and IL- $1 \beta$ upon stimulation. Here, we identify CXCL9 and CXCL10, and potentially CXCL11, as new markers of trained immunity. Moreover, our measurements of cytokines and chemokines in supernatants of recently exposed individuals as well as classical trained immunity samples revealed a number of additional candidate molecules induced during trained immunity: IL-4, CCL8 (MCP2), CCL11 (eotaxin), CCL17 (TARC), CCL19 (MIP3ß), CCL23 (MIP3), CCL25 (TECK), CCL26 (MIP4 $\alpha$ ), CCL27 (C-TACK), and CXCL13 (BLC). A considerable number of these chemokines are associated with the macrophage inflammatory response, supporting an association with trained immunity. However additional studies are required to demonstrate their functional role in trained immunity.

In conclusion, we have shown that MGIA control in human PBMCs is associated with recent Mtb infection. Control of BCG outgrowth was strongly linked to the presence of a nonclassical, CD14 ${ }^{\text {dim }}$ monocyte subset. The combination of the association with recent exposure, the temporary nature of growth reduction, and the presence of a particular monocyte subset led to the identification of trained innate immunity as the mechanism behind mycobacterial growth control. Indeed we were able to identify classical markers of trained innate immunity induced in mycobacterial control samples. In addition, we mechanistically link the CXCL10/CXCR3 axis as critical to BCG growth control as well as to trained immunity. Involvement of the CXCR3 axis may reflect the link between innate and adaptive cells involved in mycobacterial growth control. These studies have opened novel avenues to assess protective immunity against a major human pathogen, and may redirect our thinking on vaccine-induced immunity and correlates of protection in TB.

\section{Methods}

An extended version of Methods is available as supplemental information online.

Ethics and study samples. Human participation in this research was according to the US Department of Health and Human Services and Good Clinical Practice guidelines. This included protocol approval by the Leiden University Medical Center Ethics Committee and written informed consent by all donors. Anonymous buffy coats from healthy blood bank donors were used only if donors had consented scientific use of blood products. Specifically, healthy control donor samples were in vitro PPD-negative healthy buffy coats obtained from Sanquin blood transfusion services, the Netherlands. The group of healthy donors was supplemented with healthy Dutch donors before BCG vaccination, after extensive testing of their mycobacterially naive status. These donors were subsequently BCG vaccinated and analyzed at 4, 8, and 12 weeks and 1 year after BCG vaccination; the BCG vaccination study was approved with protocol P12.087 (30). A longitudinal follow-up study of individuals with LTBI was also run at Leiden University Medical Center and approved under protocol P07.048 (31). In addition, a cohort with known long-term TB latency and age-matched uninfected controls were recruited in Norway after approval by the Regional Committees for Medical and Health Research Ethics in Norway (protocol P027/99) (32, 33).
In addition, we obtained specific permission (protocol B16.002) from the ethical review board at Leiden University Medical Center to use archived samples remaining after completion of previous studies - in particular, contact investigations at a soccer club (original protocol 136/97) (34), in a supermarket (original protocol P05.053) (35-37), and among immigrants with a recent $\mathrm{TB}$ contact (original protocol P04.114) $(38,39)$.

Finally, BCG vaccination studies in healthy male volunteers were executed in Nijmegen, approved by the Arnhem-Nijmegen Medical Ethical Committee with protocol NL50160.092.24.

Experimental design. Archived PBMCs were thawed, rested, and counted. Cells were split for BCG-based MGIA as well as direct stimulation with live BCG for flow cytometry including intracellular cytokine staining. Remaining cells were dispensed in TRIzol reagents. Supernatants were collected after 4 days of coculture in the MGIA system. Upon analysis of MGIA data as well as flow cytometry data, cytokine and chemokine measurements were initiated, and functional experiments were designed.

Statistics. All data were analyzed assuming a non-Gaussian distribution, and therefore nonparametric testing was applied. All legends contain detailed information on the statistical tests applied and the number of individuals included for analysis. Generally, for comparison of groups, Kruskal-Wallis or Wilcoxon paired-rank tests were applied depending on the number of groups in the equation. For the analysis of longitudinal data, an ANOVA with Friedman test was applied. Correlations were based on linear regression modeling. All analyses were performed using GraphPad Prism version 7.00 (GraphPad Software Inc.).

\section{Author contributions}

SAJ, KEVM, and THMO designed the study, analyzed data, and wrote the manuscript. KEVM performed the experiments. SMA provided important clinical advice to the study and collected samples from various clinical cohorts. CP was responsible for sample collection in many of the clinical cohorts utilized in the study. MGN, RJWA, and RVC provided samples and contributed to study design. FO, GEK, and SVK provided clinical samples used in the study.

\section{Acknowledgments}

The authors are grateful to Louis Wilson, Department of Infectious Diseases, Leiden University Medical Center, for preparing BCG stocks for the project. This study was supported by funding from European Commission (EC) HORIZON2020 TBVAC2020 (Grant Agreement 643381); EC FP7 EURIPRED (FP7-INFRA-2012 Grant Agreement 312661); EC FP7 ADITEC (Grant Agreement 280873); and the Netherlands Organization for Scientific Research (NWO-TOP Grant Agreement 91214038). MGN was supported by a European Research Council Consolidator Grant (no. 310372) and a Spinoza Grant from the Netherlands Organization for Scientific Research. The funders had no role in study design, data collection and analysis, decision to publish, or preparation of the manuscript.

Address correspondence to: Simone A. Joosten, Department of Infectious Diseases, Leiden University Medical Center, Albinusdreef 2, 2333 ZA Leiden, the Netherlands. Phone: 31.71.5264024; Email: sajoosten@lumc.nl. 
1. Houben RM, Dodd PJ. The global burden of latent tuberculosis infection: a re-estimation using mathematical modelling. PLoS Med. 2016;13(10):e1002152.

2. World Health Organization. Global Tuberculosis Report 2016. Geneva, Switzerland: WHO; 2016. http://www.who.int/tb/publications/ global_report/gtbr2016_executive_summary.pdf. Accessed February 28, 2018.

3. Harris RC, Sumner T, Knight GM, White RG. Systematic review of mathematical models exploring the epidemiological impact of future TB vaccines. Hum Vaccin Immunother. 2016;12(11):2813-2832.

4. Abu-Raddad LJ, et al. Epidemiological benefits of more-effective tuberculosis vaccines, drugs, and diagnostics. Proc Natl Acad Sci U S A. 2009;106(33):13980-13985.

5. Ottenhoff TH, Kaufmann SH. Vaccines against tuberculosis: where are we and where do we need to go? PLoS Pathog. 2012;8(5):e1002607.

6. Ottenhoff TH, Verreck FA, Lichtenauer-Kaligis EG, Hoeve MA, Sanal O, van Dissel JT. Genetics, cytokines and human infectious disease: lessons from weakly pathogenic mycobacteria and salmonellae. Nat Genet. 2002;32(1):97-105.

7. Sakai S, et al. CD4 T cell-derived IFN- $\gamma$ plays a minimal role in control of pulmonary mycobacterium tuberculosis infection and must be actively repressed by PD-1 to prevent lethal disease. PLoS Pathog. 2016;12(5):e1005667.

8. Baguma R, et al. Application of a whole blood mycobacterial growth inhibition assay to study immunity against Mycobacterium tuberculosis in a high tuberculosis burden population. PLoS One. 2017;12(9):e0184563.

9. Fletcher HA, et al. Inhibition of mycobacterial growth in vitro following primary but not secondary vaccination with Mycobacterium bovis BCG. Clin Vaccine Immunol. 2013;20(11):1683-1689.

10. Tanner R, O'Shea MK, Fletcher HA, McShane H. In vitro mycobacterial growth inhibition assays: a tool for the assessment of protective immunity and evaluation of tuberculosis vaccine efficacy. Vaccine. 2016;34(39):4656-4665.

11. Zelmer A, et al. A new tool for tuberculosis vaccine screening: Ex vivo Mycobacterial Growth Inhibition Assay indicates BCG-mediated protection in a murine model of tuberculosis. $B M C$ Infect Dis. 2016;16:412.

12. Marsay L, et al. Mycobacterial growth inhibition in murine splenocytes as a surrogate for protection against Mycobacterium tuberculosis (M. tb). Tuberculosis (Edinb). 2013;93(5):551-557.

13. Yang AL, Schmidt TE, Stibitz S, Derrick SC, Morris SL, Parra M. A simplified mycobacterial growth inhibition assay (MGIA) using direct infection of mouse splenocytes and the MGIT system. J Microbiol Methods. 2016;131:7-9.

14. Jensen C, Lindebo Holm L, Svensson E, Aagaard C, Ruhwald M. Optimisation of a murine splenocyte mycobacterial growth inhibition assay using virulent Mycobacterium tuberculosis. Sci Rep. 2017;7(1):2830.

15. Mack U, et al. LTBI: latent tuberculosis infection or lasting immune responses to M. tuberculosis? A TBNET consensus statement. Eur Respir J. 2009;33(5):956-973.
16. Whittaker E, Kampmann B. Perinatal tuberculosis: new challenges in the diagnosis and treatment of tuberculosis in infants and the newborn. Early Hum Dev. 2008;84(12):795-799.

17. Cheon SH, et al. Bactericidal activity in whole blood as a potential surrogate marker of immunity after vaccination against tuberculosis. Clin Diagn Lab Immunol. 2002;9(4):901-907.

18. Hoft DF, et al. Investigation of the relationships between immune-mediated inhibition of mycobacterial growth and other potential surrogate markers of protective Mycobacterium tuberculosis immunity. J Infect Dis. 2002;186(10):1448-1457.

19. Worku S, Hoft DF. In vitro measurement of protective mycobacterial immunity: antigen-specific expansion of $\mathrm{T}$ cells capable of inhibiting intracellular growth of bacille Calmette-Guérin. Clin Infect Dis. 2000;30(suppl 3):S257-S261.

20. Wallis RS, et al. A whole blood bactericidal assay for tuberculosis. Jinfect Dis. 2001;183(8):1300-1303.

21. Wallis RS, et al. Whole blood bactericidal activity during treatment of pulmonary tuberculosis. J Infect Dis. 2003;187(2):270-278.

22. European Research Infrastructures for Poverty Related Diseases (EURIPRED). European Commission. http://www.euripred.eu/. Accessed March 27, 2018.

23. Brennan MJ, et al. The Cross-Species Mycobacterial Growth Inhibition Assay (MGIA) Project, 2010-2014. Clin Vaccine Immunol. 2017;24(9):e00142-17.

24. Naranbhai V, et al. Distinct transcriptional and anti-mycobacterial profiles of peripheral blood monocytes dependent on the ratio of monocytes: lymphocytes. EBioMedicine. 2015;2(11):1619-1626.

25. Smith SG, Zelmer A, Blitz R, Fletcher HA, Dockrell HM. Polyfunctional CD4 T-cells correlate with in vitro mycobacterial growth inhibition following Mycobacterium bovis BCG-vaccination of infants. Vaccine. 2016;34(44):5298-5305.

26. Tanner $\mathrm{R}$, et al. The influence of haemoglobin and iron on in vitro mycobacterial growth inhibition assays. Sci Rep. 2017;7:43478.

27. Andrews JR, Noubary F, Walensky RP, Cerda R, Losina E, Horsburgh CR. Risk of progression to active tuberculosis following reinfection with Mycobacterium tuberculosis. Clin Infect Dis. 2012;54(6):784-791.

28. Kleinnijenhuis J, et al. Bacille Calmette-Guerin induces NOD2-dependent nonspecific protection from reinfection via epigenetic reprogramming of monocytes. Proc Natl Acad Sci U S A. 2012;109(43):17537-17542.

29. Kleinnijenhuis J, et al. Long-lasting effects of BCG vaccination on both heterologous Th1/Th17 responses and innate trained immunity. J Innate Immun. 2014;6(2):152-158.

30. Boer MC, Prins C, van Meijgaarden KE, van Dissel JT, Ottenhoff TH, Joosten SA. Mycobacterium bovis BCG vaccination induces divergent proinflammatory or regulatory $\mathrm{T}$ cell responses in adults. Clin Vaccine Immunol. 2015;22(7):778-788.

31. de Paus RA, et al. Immunological characterization of latent tuberculosis infection in a low endemic country. Tuberculosis (Edinb). 2017;106:62-72.
32. Commandeur S, et al. Double- and monofunctional $\mathrm{CD}^{+}$and $\mathrm{CD}^{+} \mathrm{T}^{-}$-cell responses to Mycobacterium tuberculosis DosR antigens and peptides in long-term latently infected individuals. Eur J Immunol. 2011;41(10):2925-2936.

33. Commandeur $\mathrm{S}$, et al. Identification of human T-cell responses to Mycobacterium tuberculosis resuscitation-promoting factors in long-term latently infected individuals. Clin Vaccine Immunol. 2011;18(4):676-683.

34. Arend SM, et al. Tuberculin skin testing compared with T-cell responses to Mycobacterium tuberculosis-specific and nonspecific antigens for detection of latent infection in persons with recent tuberculosis contact. Clin Diagn Lab Immunol. 2001;8(6):1089-1096.

35. Arend SM, et al. Comparison of two interferon-gamma assays and tuberculin skin test for tracing tuberculosis contacts. Am J Respir Crit Care Med. 2007;175(6):618-627.

36. Franken WP, et al. Interferon-gamma release assays during follow-up of tuberculin skin test-positive contacts. Int J Tuberc Lung Dis. 2008;12(11):1286-1294.

37. Franken WP, et al. Follow-up study of tuberculosis-exposed supermarket customers with negative tuberculin skin test results in association with positive gamma interferon release assay results. Clin Vaccine Immunol. 2007;14(9):1239-1241.

38. Kik SV, et al. Interferon-gamma release assays in immigrant contacts and effect of remote exposure to Mycobacterium tuberculosis. Int J Tuberc Lung Dis. 2009;13(7):820-828.

39. Kik SV, et al. Predictive value for progression to tuberculosis by IGRA and TST in immigrant contacts. Eur Respir J. 2010;35(6):1346-1353.

40. Berchiche YA, Sakmar TP. CXC chemokine receptor 3 alternative splice variants selectively activate different signaling pathways. Mol Pharmacol. 2016;90(4):483-495.

41. Smith JS, et al. C-X-C motif chemokine receptor 3 splice variants differentially activate $\beta$-arrestins to regulate downstream signaling pathways. $\mathrm{Mol}$ Pharmacol. 2017;92(2):136-150.

42. Lasagni L, et al. An alternatively spliced variant of CXCR3 mediates the inhibition of endothelial cell growth induced by IP-10, Mig, and I-TAC, and acts as functional receptor for platelet factor 4 . JExp Med. 2003;197(11):1537-1549.

43. Lindestam Arlehamn CS, et al. Memory T cells in latent Mycobacterium tuberculosis infection are directed against three antigenic islands and largely contained in a CXCR $3^{+}$CCR $6^{+}$Th 1 subset. PLoS Pathog. 2013;9(1):e1003130.

44. Quintin J, et al. Candida albicans infection affords protection against reinfection via functional reprogramming of monocytes. Cell Host Microbe. 2012;12(2):223-232.

45. Matsumiya $\mathrm{M}$, et al. Inflammatory and myeloid-associated gene expression before and one day after infant vaccination with MVA85A correlates with induction of a $\mathrm{T}$ cell response. BMC Infect Dis. 2014;14:314.

46. Saha P, Geissmann F. Toward a functional characterization of blood monocytes. Immunol Cell Biol. 2011;89(1):2-4.

47. Cros J, et al. Human CD14dim monocytes patrol and sense nucleic acids and viruses via TLR7 and 
TLR8 receptors. Immunity. 2010;33(3):375-386. 48. Stenger S, et al. An antimicrobial activity of cytolytic T cells mediated by granulysin. Science. 1998;282(5386):121-125.

49. Sahiratmadja E, et al. Plasma granulysin levels and cellular interferon-gamma production correlate with curative host responses in tuberculosis, while plasma interferon-gamma levels correlate with tuberculosis disease activity in adults. Tuberculosis (Edinb). 2007;87(4):312-321.

50. Netea MG, et al. Trained immunity: a program of innate immune memory in health and disease. Science. 2016;352(6284):aaf1098.

51. Lerm M, Netea MG. Trained immunity: a new avenue for tuberculosis vaccine development. J Intern Med. 2016;279(4):337-346.

52. Tornack J, et al. Human and mouse hematopoietic stem cells are a depot for dormant Mycobacterium tuberculosis. PLoS One. 2017;12(1):e0169119.

53. Garly ML, et al. BCG scar and positive tuberculin reaction associated with reduced child mortality in West Africa. A non-specific beneficial effect of BCG? Vaccine. 2003;21(21-22):2782-2790.

54. Benn CS, Netea MG, Selin LK, Aaby P. A small jab - a big effect: nonspecific immunomodulation by vaccines. Trends Immunol. 2013;34(9):431-439.

55. Mangtani P, et al. Protection by BCG vaccine against tuberculosis: a systematic review of randomized controlled trials. Clin Infect Dis. 2014;58(4):470-480.

56. Lindestam Arlehamn CS, Sette A. Definition of CD4 immunosignatures associated with MTB. Front Immunol. 2014;5:124.

57. Kleinnijenhuis J, et al. BCG-induced trained immunity in NK cells: role for non-specific protection to infection. Clin Immunol. 2014;155(2):213-219.

58. Suliman S, et al. BCG revaccination of adults with latent Mycobacterium tuberculosis infection induces long-lived BCG-reactive natural killer cell responses. JImmunol. 2016;197(4):1100-1110. 